\title{
Article \\ Fractional N-Laplacian Problems Defined on the One-Dimensional Subspace
}

\author{
Q-Heung Choi ${ }^{1}$ (D) and Tacksun Jung ${ }^{2, *(D)}$ \\ 1 Department of Mathematics Education, Inha University, Incheon 22212, Korea; qheung@inha.ac.kr \\ 2 Department of Mathematics, Kunsan National University, Kunsan 54150, Korea \\ * Correspondence: tsjung@kunsan.ac.kr
}

check for updates

Citation: Choi, Q.-H.; Jung, T. Fractional N-Laplacian Problems Defined on the One-Dimensional Subspace. Symmetry 2021, 13, 1819. https://doi.org/10.3390/ sym13101819

Academic Editors: Calogero Vetro and Juan Luis García Guirao

Received: 23 June 2021

Accepted: 16 September 2021

Published: 29 September 2021

Publisher's Note: MDPI stays neutral with regard to jurisdictional claims in published maps and institutional affiliations.

Copyright: (c) 2021 by the authors. Licensee MDPI, Basel, Switzerland. This article is an open access article distributed under the terms and conditions of the Creative Commons Attribution (CC BY) license (https:/ / creativecommons.org/licenses/by/ $4.0 /)$.

\begin{abstract}
The research of the fractional Orlicz-Sobolev space and the fractional N-Laplacian operators will give the development of nonlinear elasticity theory, electro rheological fluids, non-Newtonian fluid theory in a porous medium as well as Probability and Analysis as they proved to be accurate models to describe different phenomena in Physics, Finance, Image processing and Ecology. We study the number of weak solutions for one-dimensional fractional N-Laplacian systems in the product of the fractional Orlicz-Sobolev spaces, where the corresponding functionals of one-dimensional fractional N-Laplacian systems are even and symmetric. We obtain two results for these problems. One result is that these problems have at least one nontrivial solution under some conditions. The other result is that these problems also have infinitely many weak solutions on the same conditions. We use the variational approach, critical point theory and homology theory on the product of the fractional Orlicz-Sobolev spaces.
\end{abstract}

Keywords: fractional N-Laplacian systems; fractional Orlicz-Sobolev spaces; product of the fractional Orlicz-Sobolev spaces; variational approach; critical point theory; homology theory

MSC: 35A01; 35A15; 35A16; 35J75

\section{Introduction}

Let $(a, b) \subset R^{1}$ and $0<s<1$ be a fractional parameter. Let $N$ denote the dimension of the domain $\Omega, N$ denote the left-hand side attached character of the $\mathrm{N}$-function and $n$ denote the dimension of the space $\left\{\left(u_{1}, \cdots, u_{n}\right)\right\} \subset R^{n}$. Let $g$ be an odd, increasing homeomorphism from $[0, \infty)$ onto $[0, \infty)$. Let $G$ be the function defined by

$$
G(x)=\int_{0}^{x} g(t) d t \quad \text { for all } x \geq 0 .
$$

$G$ is a Young function and also a N-function (The Young function $G$ is defined as follows: $G$ is the Young function if and only if $G$ satisfies that $G(0)=0, \lim _{x \rightarrow+\infty} G(x)=+\infty$ and $G$ is convex. The N-function $G$ is defined as follows: $G$ is the N-function if and only if $\lim _{x \rightarrow 0} \frac{G(x)}{x}=0, \lim _{x \rightarrow \infty} \frac{G(x)}{x}=+\infty, G(x)=0$ if and only if $\left.x=0\right)$. Let $\alpha(x), p(x)$, $m(x), \gamma(x) \in C([a, b], R)$ be measurable functions with $\alpha(x)>0,1<p(x)<\infty, m(x)>0$ and $\gamma(x)>1$. Let $D$ be an open subset in $R^{n}, n \geq 2$, with compact complement $C=R^{n} \backslash D$ containing $\theta=(0, \cdots, 0)$. Let $|\cdot|$ be denoted as a $R^{n}$ norm.

We consider the number of weak solutions $u=\left(u_{1}, \cdots, u_{n}\right) \in C^{1}([a, b], D)$ for onedimensional N-Laplacian systems with measurable positive coefficient functions 


$$
\left\{\begin{array}{cl}
(-\Delta)_{g}^{S} u_{1}(x)+\operatorname{grad}_{u_{1}}\left(\alpha(x)|u(x)|^{p(x)}\right)+\operatorname{grad}_{u_{1}}\left(m(x) \frac{1}{|u(x)|^{\gamma(x)}}\right) & =0 \text { in }(a, b), \\
\vdots & \vdots \\
(-\Delta)_{g}^{S} u_{n}(x)+\operatorname{grad}_{u_{n}}\left(\alpha(x)|u(x)|^{p(x)}\right)+\operatorname{grad}_{u_{n}}\left(m(x) \frac{1}{|u(x)|^{\gamma(x)}}\right) & =0 \text { in }(a, b) \\
u_{i}(a)=u_{i}(b), \quad u_{i}^{\prime}(a)=u_{i}^{\prime}(b) \quad \text { for all } 1 \leq i \leq n, &
\end{array}\right.
$$

where $\operatorname{grad}_{u_{i}}=\frac{\partial}{\partial u_{i}}$ and $(-\Delta)_{g}^{s} u_{i}(x)$ is the fractional N-Laplacian operator defined as follows: for each $x \in R$ and any $u_{i}$ in the fractional Orlicz-Sobolev space $W^{s} L_{G}((a, b), R)$,

$$
(-\Delta)_{g}^{s} u_{i}(x)=\text { P.V. } \int_{a}^{b} g\left(\frac{\left|u_{i}(x)-u_{i}(y)\right|}{|x-y|^{s}}\right) \frac{u_{i}(x)-u_{i}(y)}{\left|u_{i}(x)-u_{i}(y)\right|} \frac{d y}{|x-y|^{1+s}} .
$$

Here P.V. denotes the Cauchy principle value. For $0<s<1,(-\Delta)_{g}^{s}$ is called the fractional $\mathrm{N}$-Laplacian operator and for $u=\left(u_{1}, \cdots, u_{n}\right) \in W^{s} L_{G}\left((a, b), R^{n}\right)$,

$$
(-\Delta)_{g}^{S} u(x)=\left((-\Delta)_{g}^{s} u_{1}(x), \cdots,(-\Delta)_{g}^{s} u_{n}(x)\right)
$$

We assume that

(i) $\sup \left\{\left.|\alpha(x)| u(x)\right|^{p(x)}+m(x) \frac{1}{|u(x)| \gamma(x)} \mid\right.$

$\left.+\left\|\operatorname{grad}_{u}\left(\alpha(x)|u(x)|^{p(x)}\right)+\operatorname{grad}_{u}\left(m(x) \frac{1}{|u(x)|^{\gamma(x)}}\right)\right\|_{R^{n}} \mid(x, u) \in(a, b) \times\left(R^{n} \backslash B_{R_{0}}\right)\right\}<$ $+\infty$

for some $R_{0}>0$.

(ii) $\left.\quad \alpha(x)|u(x)|^{p(x)}\right)+m(x) \frac{1}{|u(x)|^{\gamma(x)}} \geq \frac{A}{d^{2}(u, C)}$ for $(x, u) \in(a, b) \times U$

for some neighborhood $U$ of $C$, the distance function $d(u, C)$ from $u$ to $C$ and a constant A $>0$.

The corresponding functionals of (1)

$$
\int_{a}^{b} \int_{a}^{b}\left[\sum_{i=1}^{n} G\left(\frac{\left|u_{i}(x)-u_{i}(y)\right|}{|x-y|^{s}}\right)\right] \frac{d x d y}{|x-y|}+\int_{a}^{b} \alpha(x)|u(x)|^{p(x)} d x+\int_{a}^{b} m(x) \frac{1}{|u|^{\gamma(x)}} d x
$$

are even and symmetric.

Our problems contain the Orlicz space, the fractional Orlicz-Sobolev space and the fractional N-Laplacian operators. The Orlicz space, the fractional Orlicz-Sobolev space and the fractional N-Laplacian operators arise in the non-linear elasticity theory, electro rheological fluids, non-Newtonian fluid theory, a porous medium and the context of stochastic Lvy processes with jumps. In recent years, Probability and Analysis in the context of Physics, Finance, Image processing and Ecology have been provided the mathematical models containing the Orlicz space, the fractional Orlicz-Sobolev space and the fractional $\mathrm{N}$-Laplacian operators to describe different phenomena.

For The Orlicz space, the fractional Orlicz-Sobolev space and the fractional N-Laplacian operators, we refer to [1-14].

For the singular elliptic systems and the singular problems involving fractional NLaplacian, we refer to [15-35].

Let $0<s<1, p: \Omega \times \Omega \rightarrow(1, \infty)$ be a continuous function with $1<p(x, y)<\infty$. The fractional Sobolev spaces with variable exponent defined on one-dimensional subspace $(a, b)$ are defined as

$$
W^{s, p(x, y)}((a, b), R)=\left\{u \in L ^ { p ( x , y ) } \left((a, b), R \mid \int_{a}^{b} \int_{a}^{b} \frac{|u(x)-u(y)|^{p(x, y)}}{\lambda^{p(x, y)}|x-y|^{1+s p(x, y)}} d x d y<\infty,\right.\right.
$$


with the norm

$$
\|u\|_{s, p(x, y)}=\inf \left\{\lambda>0 \mid \int_{a}^{b} \int_{a}^{b} \frac{|u(x)-u(y)|^{p(x, y)}}{\lambda^{p(x, y)}|x-y|^{1++s p(x, y)}} d x d y \leq 1\right\} .
$$

when $g(t)=|t|^{p(x, y)-2} t$ in (2), (2) reduced to the fractional $p(\cdot)$-Laplacian operator with variable exponent

$$
(-\Delta)_{p(\cdot)}^{s} u_{i}(x)=\text { P.V. } \int_{a}^{b} \frac{\left|u_{i}(x)-u_{i}(y)\right|^{p(x, y)-2}\left(u_{i}(x)-u_{i}(y)\right)}{|x-y|^{1+s p(x, y)}} d y, \quad x \in(a, b) .
$$

Nonsingular $p(\cdot)$-Laplacian boundary value problems like the following

$$
\begin{gathered}
-\operatorname{div}|\nabla u|^{p(x)-2} \nabla u=\lambda f(x, u), \\
u=0 \quad \partial \Omega,
\end{gathered}
$$

we refer to [2,13,36-46]. In case of $p$-Laplacian with $p(x)=p$ a constant, we refer to [3,47-63]. When $0<s<1, g(t)=t$ in (2), (2) reduced to the usual fractional Laplacian operator $(-\Delta)^{s}$. For the fractional Laplacian operator, see [45,64-87].

We deal with the fractional Orlicz spaces with variable exponent, the fractional OrliczSobolev spaces with variable exponent and their corresponding nonhomogeneous fractional NLaplacian operators as the adequate function spaces. For the theory of Orlicz and Orlicz-Sobolev spaces and the fractional N-Laplacian operator, we also refer the readers to $[1,10,60,88,89]$.

When $s=1, g(t)=|t|^{p-2} t$ and $\Omega \subset R^{n}$, (1) reduce to the $p$-Laplacian problems. We [90] proved the existence of at least one solution for the $p$-Laplacian boundary value problem with two singular and subcritical nonlinearities

$$
\left\{\begin{array}{c}
-\operatorname{div}\left(|\nabla u|^{p-2} \nabla u\right)=a|u|^{p-2} u-\operatorname{grad}_{u} \frac{1}{\left(|u|^{2}+|v|^{2}\right)^{q}}-\operatorname{grad}_{u} \frac{1}{\left(\left|u-e_{1}\right|^{2}+\left|v-e_{2}\right|^{2}\right)^{r}}+\frac{2 \alpha}{\alpha+\beta}|u|^{\alpha-1}|v|^{\beta} \\
\text { in } \Omega, \\
-\operatorname{div}\left(|\nabla v|^{p-2} \nabla v\right)=b|v|^{p-2} v-\operatorname{grad}_{v} \frac{1}{\left(|u|^{2}+|v|^{2}\right)^{q}}-\operatorname{grad}_{v} \frac{1}{\left(\left|u-e_{1}\right|^{2}+\left|v-e_{2}\right|^{2}\right)^{r}}+\frac{2 \beta}{\alpha+\beta}|u|^{\alpha}|v|^{\beta-1} \\
\text { in } \Omega,
\end{array}\right.
$$

where $a, b, p, q, r, \alpha$ and $\beta$ are real constants, and $1<p<\infty, q, r>1$ and $p<\alpha+\beta<p^{*}$, where $p^{*}$ is critical exponent such that

$$
p^{*}= \begin{cases}\frac{n p}{n-p} & \text { if } n>p, \\ \infty & \text { if } n \leq p .\end{cases}
$$

When $s=1, g(t)=|t|^{p-2} t, n=1$ and $\Omega=(a, b) \subset R^{1}$, (1) reduce to the onedimensional $p$-Laplacian problems. For the one-dimensional singular problems involving $p$-Laplacian

$$
\begin{gathered}
-\left(\left|u^{\prime}\right|^{p-2} u^{\prime}\right)^{\prime}=m(x) u^{-\gamma} \quad \text { in } \Omega, \\
u>0 \quad \text { in } \Omega, \\
u=0 \quad \text { on } \partial \Omega,
\end{gathered}
$$

we refer to $[24,30,91-101]$. For the one-dimensional fractional $p$-Laplacian problems, we refer to [102]. For the Kirchhoff equations involving fractional $p$-Laplacian, we refer to [60,76,77,103-105].

Let $G^{-1}$ be the function defined by

$$
G^{-1}(x)=\int_{0}^{x} g^{-1}(t) d t \quad \text { for all } x \geq 0 .
$$

The function $G^{-1}$ is called the complementary function of $G$ and satisfies

$$
G^{-1}(x)=\sup \{y x-G(y) \mid y \geq 0\} \quad \text { for all } x \geq 0 .
$$


$G^{-1}$ satisfies that

$$
\lim _{x \rightarrow 0} \frac{G^{-1}(x)}{x}=0, \quad \lim _{x \rightarrow \infty} \frac{G^{-1}(x)}{x}=+\infty,
$$

i.e., $G^{-1}$ is an $\mathrm{N}$-function. Moreover, by the Young's inequality,

$$
x y \leq G(x)+G^{-1}(y), \quad \text { for all } x, y \geq 0 .
$$

From now on we shall denote by $C^{1}\left(S^{1}, D\right)$ the subset of $C^{1}([a, b], D)$ satisfying the conditions

$$
u(a)=u(b), \quad u^{\prime}(a)=u^{\prime}(b) .
$$

Orlicz space $L_{G}\left(S^{1}, R\right)$ defined by $\mathrm{N}$-function $G$ is defined as

$$
\begin{aligned}
L_{G}\left(S^{1}, R\right) & =\left\{u \mid u: S^{1} \rightarrow R\right. \text { is a measurable function with } \\
\|u\|_{L_{G}} & \left.=\sup \left\{\int_{a}^{b} u v d x \mid \int_{a}^{b} G^{-1}(|v|) d x \leq 1\right\}<\infty\right\} .
\end{aligned}
$$

$L_{G}\left(S^{1}, R\right)$ is a Banach space with a norm $\|u\|_{L_{G}}$ equivalent to the Luxemburg norm

$$
\|u\|_{G}=\inf \left\{v>0 \mid \int_{a}^{b} G\left(\left|\frac{u(x)}{v}\right|\right) \leq 1\right\} .
$$

Hölder inequality holds in the Orlicz space $L_{G}\left(S^{1}, R\right)$ (see [19]):

$$
\int_{a}^{b}|u v| d x \leq 2\|u\|_{L_{G}}\|v\|_{L_{G^{-1}}} \quad \text { for all } u, v \in L_{G}\left(S^{1}, R\right) .
$$

Orlicz-Sobolev space $W^{s} L_{G}\left(S^{1}, R\right)$ is defined as

$$
W^{s} L_{G}\left(S^{1}, R\right)=\left\{u \in L_{G}\left(S^{1}, R\right): \int_{a}^{b} \int_{a}^{b} G\left(\frac{|u(x)-u(y)|}{|x-y|^{s}}\right) \frac{d x d y}{|x-y|}<\infty\right\}
$$

with the norm

$$
\|u\|_{s, G}=\|u\|_{G}+[u]_{s, G},
$$

where $[u]_{s, G}$ is the Gagliardo semi-norm defined by

$$
[u]_{s, G}=\inf \left\{\mu>0 \mid \int_{a}^{b} \int_{a}^{b} G\left(\frac{|u(x)-u(y)|}{\mu|x-y|^{s}}\right) \frac{d x d y}{|x-y|} \leq 1\right\} .
$$

In [88], for any $0<s<1$ and $G$ a Young function such that $G$ and $G^{-1}$ satisfy that

$$
G(2 t) \leq C_{1} G(t) \text { and } G^{-1}(2 t) \leq C_{2} G^{-1}(t), \quad \forall t \geq 0, C_{1}, C_{2}>0,
$$

$W^{s} L_{G}\left(S^{1}, R\right)$ is reflexive and separable. Moreover $C_{0}^{\infty}\left(S^{1}, R\right)$ is dense in $W^{s} L_{G}\left(S^{1}, R\right)$ in the norm $\|\cdot\|_{s, G}$. Let the Orlicz-Sobolev space $W_{0}^{s} L_{G}\left(S^{1}, R\right)$ be the closure of $C_{0}^{\infty}\left(S^{1}, R\right)$ in $W^{s} L_{G}\left(S^{1}, R\right)$. The space $W_{0}^{s} L_{G}\left(S^{1}, R\right)$ is also reflexive. By Lemma 5.7 in [4], the norm $\|u\|_{s, G}$ is equivalent to $[u]_{s, G}$.

Let

$$
L_{G}\left(S^{1}, R^{n}\right)=L_{G}\left(S^{1}, R\right) \times \cdots \times L_{G}\left(S^{1}, R\right)
$$

be the $n$-cartesian product space of $L_{G}\left(S^{1}, R\right)$ equipped with the norm

$$
\|u\|_{L_{G}\left(S^{1}, R^{n}\right)}=\sum_{i=1}^{n}\left\|u_{i}\right\|_{L_{G}\left(S^{1}, R\right)}, \quad u=\left(u_{1}, \cdots, u_{n}\right)
$$

and

$$
H=W^{s} L_{G}\left(S^{1}, R^{n}\right)=W^{s} L_{G}\left(S^{1}, R\right) \times \cdots \times W^{s} L_{G}\left(S^{1}, R\right)
$$


equipped with the norm

$$
\|u\|_{H}=\sum_{i=1}^{n}\left\|u_{i}\right\|_{s, G}
$$

For any $G$ a Young function such that $G$ and $G^{-1}$ satisfy $(5), W^{s} L_{G}\left(S^{1}, R^{n}\right)$ is reflexive and separable. Moreover $C_{0}^{\infty}\left(S^{1}, R^{n}\right)$ is dense in $W^{s} L_{G}\left(S^{1}, R^{n}\right)$ in the norm $\|\cdot\|_{s, G}$. Let $W_{0}^{s} L_{G}\left(S^{1}, R^{n}\right)$ be the closure of $C_{0}^{\infty}\left(S^{1}, R^{n}\right)$ in $W^{s} L_{G}\left(S^{1}, R^{n}\right)$.

Zhikov [106] observed that smooth functions are not dense in $W^{1, p(x)}\left(S^{1}, R\right)$ without additional assumptions on the exponent $p(x)$. However, when the exponent $p(x)$ is logHölder continuous, i.e., there is a constant $B$ such that

$$
|p(x)-p(y)| \leq \frac{B}{-\log |x-y|}
$$

for every $x, y \in(a, b)$ with $|x-y| \leq \frac{1}{2}$, then smooth functions are dense in $W^{1, p(x)}\left(S^{1}, R\right)$. and there is no confusion in defining the Sobolev space with zero boundary values, $W_{0}^{1, p(x)}$, as the completion of $C_{0}^{\infty}\left(S^{1}, R\right)$ with respect to the norm $\|u\|_{W^{1, p(x)}\left(S^{1}, R\right)}[39,93]$.

Let us set

$$
g_{0}=\inf _{t>0} \frac{\operatorname{tg}(t)}{G(t)} \quad g^{0}=\sup _{t>0} \frac{\operatorname{tg}(t)}{G(t)}
$$

and assume that

$$
1<g_{0} \leq \frac{\operatorname{tg}(t)}{G(t)} \leq g^{0}<\infty \quad \forall t \geq 0 .
$$

By Proposition 2.3 of [6], it implies that each $G$ satisfies the $\Delta_{2}$-condition, i.e., there exists a constant $C>0$ such that

$$
G(2 t) \leq C G(t), \quad t \geq 0 .
$$

Assume that $G$ is a function such that

$$
G: t \in[0, \infty) \mapsto G(\sqrt{t}) \text { is convex. }
$$

Weak solutions $u=\left(u_{1}, \cdots, u_{n}\right) \in W^{s} L_{G}\left(S^{1}, D\right)$ satisfy

$\int_{a}^{b}\left[(-\Delta)_{g}^{s} u(x) \cdot \phi(x) d x+\operatorname{grad}_{u}\left(\alpha(x)|u(x)|^{p(x)}\right) \cdot \phi(x)+\operatorname{grad}_{u}\left(m(x) \frac{1}{|u|^{\gamma(x)}}\right) \cdot \phi(x)\right] d x=0$

$\forall \phi \in W^{s} L_{G}\left(S^{1}, D\right)$.

Theorem 1. Let $0<s<1, s g_{0}<1, p \in C([a, b], R)$ with $p(x)>1$ be log-Hölder continuous. Let $\alpha(x), p(x), m(x), \gamma(x) \in C([a, b], R)$ be measurable functions with $\alpha(x)>0,1<p(x)<\infty$, $m(x)>0$ and $\gamma(x)>1$. We assume that (5)-(7) and

$$
1<p(x) \leq p_{+}<g_{0}^{*}=\frac{g_{0}}{1-s g_{0}}
$$

hold, where $p_{+}=\sup _{x \in[a, b]} p(x)$ and $p_{-}=\inf _{x \in[a, b]} p(x)$. Then (1) has at least one nontrivial weak solution.

Theorem 2. Under the assumptions of Theorem 1, (1) has infinitely many weak solutions.

We use variational approach, minimax method in critical point theory on the loop space $W^{s} L_{G}\left(S^{1}, D\right)$ and homology theory. In Section 2, we introduce some preliminaries. In Section 3, we obtain some variational results on the potential and prove that the associated functional $J$ of (1) satisfies the (P.S.) condition on the loop subspace $W^{s} L_{G}\left(S^{1}, D\right)$. In Section 4 , we prove Theorems 1 and 2 by using minimax method, critical point theory and homology theory. 


\section{Fractional Orlicz-Sobolev Space}

For the variational setting, we need some properties on $L^{p(x)}\left(S^{1}, R\right), L^{p(x)}\left(S^{1}, R^{n}\right)$, $L_{G}\left(S^{1}, R\right), L_{G}\left(S^{1}, R^{n}\right), W^{s} L_{G}\left(S^{1}, R\right)$ and $W^{s} L_{G}\left(S^{1}, R^{n}\right)$ which are introduced in Section 1 and can be found in $[107,108]$.

Lemma 1. ([107]) The space $L^{p(x)}\left(S^{1}, R\right)$ is a separable, uniformly convex Banach space, and its conjugate space is $L^{p^{\prime}(x)}\left(S^{1}, R\right)$, where $\frac{1}{p(x)}+\frac{1}{p^{\prime}(x)}=1$. Let $u \in L^{p(x)}\left(S^{1}, R\right)$ and $v \in$ $L^{p^{\prime}(x)}\left(S^{1}, R\right)$. Then we have

$$
\left|\int_{a}^{b} u v d x\right| \leq\left(\frac{1}{p_{-}}+\frac{1}{\left(p^{\prime}\right)_{-}}\right)\|u\|_{L^{p(x)}\left(S^{1}, R\right)}\|v\|_{L^{p^{\prime}(x)}\left(S^{1}, R\right)} \leq 2\|u\|_{L^{p(x)}\left(S^{1}, R\right)}\|v\|_{L^{p^{\prime}(x)}\left(S^{1}, R\right)} .
$$

Lemma 2. Let $p_{+}<\infty$ and set

$$
\tau(u)=\int_{a}^{b}|u|^{p(x)} d x \quad \text { for all } u \in L^{p(x)}\left(S^{1}, R\right) .
$$

Then

(i) $\begin{aligned} \tau(u)>1 & \Leftrightarrow\|u\|_{L^{p(x)}\left(S^{1}, R\right)}>1, \\ \tau(u)=1 & \Leftrightarrow\|u\|_{L^{p(x)}\left(S^{1}, R\right)}=1, \\ \tau(u)<1 & \Leftrightarrow\|u\|_{L^{p(x)}\left(S^{1}, R\right)}<1 .\end{aligned}$

(ii) If $\|u\|_{L^{p(x)}\left(S^{1}, R\right)}>1$, then $\|u\|_{L^{p(x)}\left(S^{1}, R\right)}^{p_{-}} \leq \tau(u) \leq\|u\|_{L^{p(x)}\left(S^{1}, R\right)}^{p_{+}}$.

(iii) If $\|u\|_{L^{p(x)}\left(S^{1}, R\right)}<1$, then $\|u\|_{L^{p(x)}\left(S^{1}, R\right)}^{p_{+}} \leq \tau(u) \leq\|u\|_{L^{p(x)}\left(S^{1}, R\right)}^{\left.p^{(}\right)}$.

Lemma 3. ([88]) Let $0<s<1$ and $G$ be a $N$-function. Then $W^{s} L_{G}\left(S^{1}, R^{n}\right)$ is a reflexive and separable Banach space. Furthermore $C_{0}^{\infty}\left(S^{1}, R^{n}\right)$ is dense in $W^{s} L_{G}\left(S^{1}, R^{n}\right)$ in the norm $\|\cdot\|_{s, G}$.

Let $u_{i} \in W^{s} L_{G}\left(S^{1}, R\right)$ and set

$$
\left[u_{i}\right]_{s, G}=\inf \left\{\sigma>0 \mid \int_{a}^{b} \int_{a}^{b} G\left(\frac{\left|u_{i}(x)-u_{i}(y)\right|}{\sigma|x-y|^{s}}\right) \frac{d x d y}{|x-y|} \leq 1\right\}, \quad 1 \leq i \leq n .
$$

Lemma 4. ([109]) (Generalized Poincar inequality on the Orlicz-Sobolev space) Let $0<s<1$ and $G$ be a Young function. Then

$$
\left\|u_{i}\right\|_{G} \leq v_{i}\left[u_{i}\right]_{s, G}, \quad \forall u_{i} \in W^{s} L_{G}\left(S^{1}, R\right), \quad 1 \leq i \leq n
$$

for some constants $v_{i}>0,1 \leq i \leq n$. That is, the embedding

$$
W^{s} L_{G}\left(S^{1}, R\right) \hookrightarrow L_{G}\left(S^{1}, R\right)
$$

is continuous. Furthermore $\left[u_{i}\right]_{s, G}$ is a norm of $W^{s} L_{G}\left(S^{1}, R\right)$ equivalent to $\left\|u_{i}\right\|_{s, G}$.

Let $u=\left(u_{1}, \cdots, u_{n}\right) \in W^{s} L_{G}\left(S^{1}, R^{n}\right), u_{i} \in W^{s} L_{G}\left(S^{1}, R\right)$ and set

$$
[u]_{s, G}=\sum_{i=1}^{n}\left[u_{i}\right]_{s, G} .
$$

Combining Lemma 4 and (9), we obtain the following lemma:

Lemma 5. (Generalized Poincar inequality on the product of the Orlicz-Sobolev space) Let $0<s<1$ and $G$ be a Young function. Then

$$
\|u\|_{G} \leq v[u]_{s, G}, \quad \forall u \in W^{s} L_{G}\left(S^{1}, R^{n}\right)
$$


for some constant $v>0$. That is, the embedding

$$
W^{s} L_{G}\left(S^{1}, R^{n}\right) \hookrightarrow L_{G}\left(S^{1}, R^{n}\right)
$$

is continuous. Moreover $[u]_{s, G}$ is a norm of $W^{s} L_{G}\left(S^{1}, R^{n}\right)$ equivalent to $\|\cdot\|_{s, G}$.

Lemma 6. ([110]) Let $u_{i} \in W^{s} L_{G}\left(S^{1}, R\right)$. Then

$$
\begin{aligned}
\left\|u_{i}\right\|_{s, G}^{g_{0}} & \leq \int_{a}^{b} \int_{a}^{b} G\left(\frac{\left|u_{i}(x)-u_{i}(y)\right|}{|x-y|^{s}}\right) \frac{d x d y}{|x-y|} \leq\left\|u_{i}\right\|_{s, G}^{g^{0}}, \text { if }\left\|u_{i}\right\|_{s, G}>1,1 \leq i \leq n,(10) \\
\left\|u_{i}\right\|_{s, G}^{g^{0}} & \leq \int_{a}^{b} \int_{a}^{b} G\left(\frac{\left|u_{i}(x)-u_{i}(y)\right|}{|x-y|^{s}}\right) \frac{d x d y}{|x-y|} \leq\left\|u_{i}\right\|_{s, G^{\prime}}^{g_{0}} \text { if }\left\|u_{i}\right\|_{s, G}<1 .
\end{aligned}
$$

Proof. The proof follows from (6) and Theorem 3.11 of [110].

Let us define the functional $Q_{s, G}: W^{s} L_{G}\left(S^{1}, R^{n}\right) \rightarrow R$ by

$$
\begin{gathered}
Q_{s, G}(u)=\int_{a}^{b} \int_{a}^{b}\left[\sum_{i=1}^{n} G\left(\frac{\left|u_{i}(x)-u_{i}(y)\right|}{|x-y|^{s}}\right)\right] \frac{d x d y}{|x-y|^{\prime}}, \\
u=\left(u_{1}, \cdots, u_{n}\right) \in W^{s} L_{G}\left(S^{1}, R^{n}\right), u_{i} \in W^{s} L_{G}\left(S^{1}, R\right) .
\end{gathered}
$$

Combining Lemma 6 and (9), we obtain that:

Lemma 7. Let $u \in W^{s} L_{G}\left(S^{1}, R^{n}\right)$. Then

$$
\begin{aligned}
& \|u\|_{s, G}^{g_{0}} \leq Q_{s, G}(u) \leq\|u\|_{s, G^{\prime}}^{g^{0}}, \quad \text { if }\|u\|_{s, G}>1, \\
& \|u\|_{s, G}^{g^{0}} \leq Q_{s, G}(u) \leq\|u\|_{s, G^{\prime}}^{g_{0}}, \quad \text { if }\|u\|_{s, G}<1 .
\end{aligned}
$$

Lemma 8. ([109]) Let $0<s<1, s g_{0}<1$ and $G$ be a $N$-function. Then the embeddings

$$
W^{s, g_{0}}\left(S^{1}, R\right) \hookrightarrow L^{r(x)}\left(S^{1}, R\right)
$$

and

$$
W^{s} L_{G}\left(S^{1}, R\right) \hookrightarrow L^{r(x)}\left(S^{1}, R\right)
$$

are continuous and compact for all $1 \leq r(x)<g_{0}^{*}$.

Moreover

$$
\left\|u_{i}\right\|_{L^{r(x)}\left(S^{1}, R\right)} \leq C_{i}\left[u_{i}\right]_{s, G} \text { for all } 1 \leq r(x)<g_{0}^{*}
$$

for some constants $C_{i}, 1 \leq i \leq n$.

Proof. By [109], the embedding $W^{s, g_{0}}\left(S^{1}, R\right) \hookrightarrow L^{r(x)}\left(S^{1}, R\right)$ is continuous and compact for all $1 \leq r(x)<g_{0}^{*}$. By (6), the embedding $W^{s} L_{G}\left(S^{1}, R\right) \hookrightarrow W^{s, g_{0}}\left(S^{1}, R\right)$ is continuous. Combining these facts, we obtain that the embedding $W^{s} L_{G}\left(S^{1}, R\right) \hookrightarrow L^{r(x)}\left(S^{1}, R\right)$ is continuous and compact for all $1 \leq r(x)<g_{0}^{*}$.

Lemma 9. Let $0<s<1, s g_{0}<1$ and $G$ be a $N$-function. Then the embeddings

$$
W^{s, g_{0}}\left(S^{1}, R^{n}\right) \hookrightarrow L^{r(x)}\left(S^{1}, R^{n}\right)
$$

and

$$
W^{s} L_{G}\left(S^{1}, R^{n}\right) \hookrightarrow L^{r(x)}\left(S^{1}, R^{n}\right)
$$

are continuous and compact for all $1 \leq r(x)<g_{0}^{*}$.

Moreover

$$
\|u\|_{L^{r(x)}\left(S^{1}, R^{n}\right)} \leq C[u]_{S, G} \text { for all } 1 \leq r(x)<g_{0}^{*} .
$$


for some constant $C>0$.

Lemma 10. Weakly convergent sequence $\left\{u_{k}=\left(u_{k_{1}}, \cdots, u_{k_{n}}\right)\right\}$ converging to $u=\left(u_{1}, \cdots, u_{n}\right)$ in $W^{s} L_{G}\left(S^{1}, R^{n}\right)$ satisfying

$$
\lim _{k \rightarrow+\infty} \sup <Q_{s, G}^{\prime}\left(u_{k}\right), u_{k}-u>\leq 0 .
$$

converges strongly to $u$ in $W^{s} L_{G}\left(S^{1}, R^{n}\right)$.

Proof. Since the sequence $\left\{u_{k}\right\}$ converges weakly to $u$ in $W^{s} L_{G}\left(S^{1}, R^{n}\right)$ and $\lim _{k \rightarrow+\infty} \sup <Q^{\prime}\left(u_{k}\right), u_{k}-u>\leq 0$, by (5), we have

$$
\begin{aligned}
& \int_{a}^{b} \int_{a}^{b}\left[\sum_{i=1}^{n} g\left(\frac{\left|u_{k_{i}}(x)-u_{k_{i}}(y)\right|}{|x-y|^{s}}\right) \frac{u_{k_{i}}(x)-u_{k_{i}}(y)}{\left|u_{k_{i}}(x)-u_{k_{i}}(y)\right|} \frac{u_{k_{i}}(x)-u_{k_{i}}(y)}{|x-y|^{s}}\right] \frac{d x d y}{|x-y|} \\
& \leq \int_{a}^{b} \int_{a}^{b}\left[\sum_{i=1}^{n} g\left(\frac{\left|u_{i}(x)-u_{i}(y)\right|}{|x-y|^{s}}\right) \frac{u_{i}(x)-u_{i}(y)}{\left|u_{i}(x)-u_{i}(y)\right|} \frac{u_{i}(x)-u_{i}(y)}{|x-y|^{s}}\right] \frac{d x d y}{|x-y|} \\
& \leq g^{0}\left[\int_{a}^{b} \int_{a}^{b}\left[\sum_{i=1}^{n} G\left(\frac{\left|u_{i}(x)-u_{i}(y)\right|}{|x-y|^{s}}\right)\right] \frac{d x d y}{|x-y|} .\right.
\end{aligned}
$$

Sequence $\left\{\int_{a}^{b} \int_{a}^{b}\left[\sum_{i=1}^{n} g\left(\frac{\left|u_{k_{i}}(x)-u_{k_{i}}(y)\right|}{|x-y|^{s}}\right) \frac{u_{k_{i}}(x)-u_{k_{i}}(y)}{\left|u_{k_{i}}(x)-u_{k_{i}}(y)\right|} \frac{u_{k_{i}}(x)-u_{k_{i}}(y)}{|x-y|^{s}}\right] \frac{d x d y}{|x-y|}\right\}$ is bounded and converges to

$\int_{a}^{b} \int_{a}^{b}\left[\sum_{i=1}^{n} g\left(\frac{\left|u_{i}(x)-u_{i}(y)\right|}{|x-y|^{\mathrm{s}}}\right) \frac{u_{i}(x)-u_{i}(y)}{\left|u_{i}(x)-u_{i}(y)\right|} \frac{u_{i}(x)-u_{i}(y)}{|x-y|^{\mathrm{s}}}\right] \frac{d x d y}{|x-y|}$. By (6), we have

$$
\begin{aligned}
& \int_{a}^{b} \int_{a}^{b}\left[\sum_{i=1}^{n} g\left(\frac{\left|u_{k_{i}}(x)-u_{k_{i}}(y)\right|}{|x-y|^{s}}\right) \frac{u_{k_{i}}(x)-u_{k_{i}}(y)}{\left|u_{k_{i}}(x)-u_{k_{i}}(y)\right|} \frac{u_{k_{i}}(x)-u_{k_{i}}(y)}{|x-y|^{s}}\right] \frac{d x d y}{|x-y|} \\
& \geq g_{0}\left[\int_{a}^{b} \int_{a}^{b}\left[\sum_{i=1}^{n} G\left(\frac{\left|u_{k_{i}}(x)-u_{k_{i}}(y)\right|}{|x-y|^{s}}\right)\right] \frac{d x d y}{|x-y|} .\right.
\end{aligned}
$$

Sequence $\left\{\int_{a}^{b} \int_{a}^{b}\left[\sum_{i=1}^{n} G\left(\frac{\left|u_{k_{i}}(x)-u_{k_{i}}(y)\right|}{|x-y|^{s}}\right)\right] \frac{d x d y}{|x-y|}\right\}$ is bounded and converges to $\int_{a}^{b} \int_{a}^{b}\left[\sum_{i=1}^{n} G\left(\frac{\left|u_{i}(x)-u_{i}(y)\right|}{|x-y|^{s}}\right)\right] \frac{d x d y}{|x-y|}$. Thus the sequence $\left\{u_{k}\right\}$ is bounded and converges weakly to $u$ in $W^{s} L_{G}\left(S^{1}, R^{n}\right)$. Since the embedding $W^{s} L_{G}\left(S^{1}, R^{n}\right) \hookrightarrow L_{G}\left(S^{1}, R^{n}\right)$ is continuous and compact, $\left\{u_{k}\right\}$ converges strongly to $u$ in $W^{s} L_{G}\left(S^{1}, R^{n}\right)$.

Lemma 11. If $u_{k}=\left(u_{k_{1}}, \cdots, u_{k_{n}}\right), u=\left(u_{1}, \cdots, u_{n}\right) \in W^{s} L_{G}\left(S^{1}, R^{n}\right), k=1,2, \ldots$, then the following statement are equivalent to each other

(i) $\lim _{k \rightarrow \infty}\left\|u_{k}-u\right\|_{s, G}=0, k=1,2$,

(ii) $\lim _{k \rightarrow \infty} \int_{a}^{b}\left[\sum_{i=1}^{n}\left(G\left(u_{k_{i}}(x)-u_{i}(x)\right)\right] d x=0\right.$ and

$\lim _{k \rightarrow \infty}\left[u_{k}-u\right]_{s, G}=0$,

(iii) $u_{k} \rightarrow u$ in measure in $W^{s} L_{G}\left(S^{1}, R^{n}\right)$ and

$\lim _{k \rightarrow \infty} \int_{a}^{b}\left[\sum_{i=1}^{n} G\left(u_{k_{i}}(x)\right)\right] d x=\int_{a}^{b}\left[\sum_{i=1}^{n} G\left(u_{i}(x)\right)\right] d x$.

Proof. By the definition of $\|\cdot\|_{s, G},(\mathrm{i}) \Leftrightarrow$ (ii) holds. We shall show that (i) implies (iii). We assume that (i) holds. Then

$$
\begin{aligned}
& \int_{a}^{b}\left[\left(\sum_{i=1}^{n} G\left(u_{k_{i}}\right)(x)\right)-\left(\sum_{i=1}^{n} G\left(u_{i}\right)(x)\right)\right] d x \\
& \leq \int_{a}^{b}\left[\sum_{i=1}^{n}\left(g\left(u_{i}+\lambda\left(u_{k_{i}}-u_{i}\right)\right)\left(u_{k_{i}}-u_{i}\right)\right)\right] d x \\
& \leq 2\left[\sum_{i=1}^{n}\left(\left\|g\left(u_{i}+\lambda\left(u_{k_{i}}-u_{i}\right)\right)\right\|_{G^{-1}}\left\|u_{k_{i}}-u_{i}\right\|_{G}\right)\right] \\
& \leq C\left(\sum_{i=1}^{n}\left(\left\|g\left(u_{i}+\lambda\left(u_{k_{i}}-u_{i}\right)\right)\right\|_{G^{-1}}\left\|u_{k_{i}}-u_{i}\right\|_{s, G}\right)\right) \\
& \rightarrow 0
\end{aligned}
$$


for $0<\lambda<1$ and some $C>0$. It follows that (iii) holds. Assume that (iii) holds. Since $\lim _{k \rightarrow \infty} \int_{a}^{b}\left[\sum_{i=1}^{n} G\left(u_{k_{i}}(x)\right)\right] d x=\int_{a}^{b}\left[\sum_{i=1}^{n} G\left(u_{i}(x)\right)\right] d x,\left\{u_{k}\right\}$ converges weakly to $u$ in $L_{G}\left(S^{1}\right.$, $\left.R^{n}\right)$. By assumption (iii), $u_{k} \rightarrow u$ in measure in $W^{s} L_{G}\left(S^{1}, R^{n}\right)$. It follows that $\left\{u_{k}\right\}$ is bounded in $W^{s} L_{G}\left(S^{1}, R^{n}\right) \subset L_{G}\left(S^{1}, R^{n}\right)$. By Lemma 9, the embedding $W^{s} L_{G}\left(S^{1}, R^{n}\right) \hookrightarrow$ $L_{G}\left(S^{1}, R^{n}\right)$ is continuous and compact. Thus $u_{k} \rightarrow u$ strongly in $W^{s} L_{G}\left(S^{1}, R^{n}\right)$. Thus (i) holds.

\section{Variational Results}

Let us set an open set of the Hilbert space $W^{s} L_{G}\left(S^{1}, R^{n}\right)$ as

$$
\begin{gathered}
\Lambda_{s, G} D=\left\{u \in W^{s} L_{G}\left(S^{1}, R^{n}\right) \mid \int_{a}^{b} \int_{a}^{b}\left[\sum_{i=1}^{n} G\left(\frac{\left|u_{i}(x)-u_{i}(y)\right|}{|x-y|^{s}}\right)\right] \frac{d x d y}{|x-y|}<\infty,\right. \\
\left.\forall u(x)=\left(u_{1}(x), \cdots, u_{n}(x)\right) \in D=R^{n} \backslash C \subset R^{n}, \forall x \in S^{1}, \theta \in C, \theta=(0, \cdots, 0)\right\}
\end{gathered}
$$

the loop space on $D$. We note that $\operatorname{grad}_{u}\left(\frac{m(x)}{|u(x)| \gamma(x)}\right)$ is not singular on $W^{s} L_{G}\left(S^{1}, D\right)$ because $D$ is away from 0 .

Let us define the functional $J_{s, G}: \Lambda D \subset W^{s} L_{G}\left(S^{1}, R^{n}\right) \rightarrow R$ by

$$
J_{s, G}(u)=Q_{s, G}(u)+\int_{a}^{b} \alpha(x)|u(x)|^{p(x)} d x+\int_{a}^{b} m(x) \frac{1}{|u| \gamma(x)} d x,
$$

where

$$
Q_{s, G}(u)=\int_{a}^{b} \int_{a}^{b}\left[\sum_{i=1}^{n} G\left(\frac{\left|u_{i}(x)-u_{1}(y)\right|}{|x-y|^{s}}\right)\right] \frac{d x d y}{|x-y|}
$$

for $u=\left(u_{1}, \cdots, u_{n}\right) \in \Lambda D$. Then the functional $Q_{s, G}$ is of class $C^{1}\left(W^{s} L_{G}\left(S^{1}, R^{n}\right), R\right)$ with

$$
\begin{aligned}
& <Q_{s, G}^{\prime}(u), v>=<(-\Delta)_{g}^{s} u, v> \\
& =\int_{a}^{b} \int_{a}^{b}\left[\sum_{i=1}^{n} g\left(\frac{\left|u_{i}(x)-u_{i}(y)\right|}{|x-y|^{s}}\right) \frac{u_{i}(x)-u_{i}(y)}{\left|u_{i}(x)-u_{i}(y)\right|} \frac{v_{i}(x)-v_{i}(y)}{|x-y|^{s}}\right] \frac{d x d y}{|x-y|}
\end{aligned}
$$

$\forall u=\left(u_{1}, \cdots, u_{n}\right) \in \Lambda D$, which is proved in Proposition 3.3 in [89].

Lemma 12. $J_{s, G}(u)$ is continuous and $C^{1}$ on $\Lambda_{s, G} D$ with Frchet derivative

$$
\begin{aligned}
& D J_{s, G}(u) v \\
& =\int_{a}^{b} \int_{a}^{b}\left[\sum_{i=1}^{n} g\left(\frac{\left|u_{i}(x)-u_{i}(y)\right|}{|x-y|^{s}}\right) \frac{u_{i}(x)-u_{i}(y)}{\left|u_{i}(x)-u_{i}(y)\right|} \frac{v_{i}(x)-v_{i}(y)}{|x-y|^{s}}\right] \frac{d x d y}{|x-y|} \\
& \quad+\int_{a}^{b}\left[\left(\operatorname{grad}_{u} \alpha(x)|u(x)|^{p(x)}\right)\right) \cdot v(x)+\left(\operatorname{grad}_{u}\left(\frac{m(x)}{|u(x)|^{\gamma(x)}}\right) \cdot v(x)\right] d x,
\end{aligned}
$$

$\forall v=\left(v_{1}, \cdots, v_{n}\right) \in \Lambda_{s, G}$ D. Moreover $D J_{s, G} \in C$. That is, $J_{s, G} \in C^{1}$.

Proof. By Proposition 3.3 in [89],

$$
Q_{s, G}(u)=\int_{a}^{b} \int_{a}^{b}\left[\sum_{i=1}^{n} G\left(\frac{\left|u_{i}(x)-u_{i}(y)\right|}{|x-y|^{s}}\right)\right] \frac{d x d y}{|x-y|}
$$

is $C^{1}$ with Frchet derivative

$$
\begin{aligned}
<Q_{s, G}^{\prime}(u), v> & =\left\langle(-\Delta)_{g}^{s} u, v>\right. \\
& =\int_{a}^{b} \int_{a}^{b}\left[\sum_{i=1}^{n} g\left(\frac{\left|u_{i}(x)-u_{i}(y)\right|}{|x-y|^{s}}\right) \frac{u_{i}(x)-u_{i}(y)}{\left|u_{i}(x)-u_{i}(y)\right|} \frac{v_{i}(x)-v_{i}(y)}{|x-y|^{s}}\right] \frac{d x d y}{|x-y|} .
\end{aligned}
$$


It suffices to show that $\int_{a}^{b} \alpha(x)|u(x)|^{p(x)} d x+\int_{a}^{b} \frac{m(x)}{|u(x)| \gamma(x)} d x$ is $C^{1}$ with Frchet derivative $\int_{a}^{b}\left(\operatorname{grad}_{u} \alpha(x)|u(x)|^{p(x)}\right) \cdot v(x)+\int_{a}^{b}\left(\operatorname{grad}_{u}\left(\frac{m(x)}{|u(x)| \gamma(x)}\right) \cdot v(x) d x \forall v \in \Lambda_{s, G} D\right.$. Let us set

$$
\Psi(u)=\Psi_{1}(u)+\Psi_{2}(u),
$$

where

$$
\Psi_{1}(u)=\int_{a}^{b} \alpha(x)|u(x)|^{p(x)} d x, \quad \Psi_{2}(u)=\int_{a}^{b} \frac{m(x)}{|u(x)| \gamma(x)} d x .
$$

We shall prove that $\Psi(u)$ is continuous. For $v, w \in \Lambda_{s, G} D$, we have

$$
\begin{aligned}
& \left|\Psi_{1}(v+w)-\Psi_{1}(v)\right| \\
& \leq|\alpha(x)|_{\infty}\left|\int_{a}^{b}\left[\left(\operatorname{grad}_{v}\left|v(x)+\tau_{1} w(x)\right|^{p(x)}\right) \cdot w(x)+O\left(\|w\|_{H}\right)\right] d x\right|=O\left(\|w\|_{H}\right)
\end{aligned}
$$

for some $0<\tau_{1}<1$. and

$$
\begin{aligned}
& \left|\Psi_{2}(v+w)-\Psi_{2}(v)\right| \\
& \leq|m(x)|_{\infty}\left|\int_{a}^{b}\left[\left(\operatorname{grad}_{v} \frac{1}{\left|v(x)+\tau_{2} w(x)\right| \gamma(x)}\right) \cdot w(x)+O\left(\|w\|_{H}\right)\right] d x\right|=O\left(\|w\|_{H}\right)
\end{aligned}
$$

for some $0<\tau_{2}<1$. Thus we have

$|\Psi(v+w)-\Psi(v)| \leq\left|\Psi_{1}(v+w)-\Psi_{1}(v)\right|+\left|\Psi_{2}(v+w)-\Psi_{2}(v)\right|=O\left(\|w\|_{H}\right)$. Next we shall prove that $\Psi(u)$ is $C^{1}$ in $\Lambda_{s, G} D$. Let $v, w \in \Lambda_{s, G} D$. By (14) and (15), we have

$$
\left|\Psi_{1}(v+w)-\Psi_{1}(v)-D \Psi_{1}\left(v+\tau_{1} w\right) \cdot w\right|=0\left(\|w\|_{H}\right) .
$$

for some $\tau_{1}>0$ and

$$
\left|\Psi_{1}(v+w)-\Psi_{2}(v)-D \Psi_{1}\left(v+\tau_{2} w\right) \cdot w\right|=0\left(\|w\|_{H}\right) .
$$

for some $\tau_{2}>0$. Thus $\Psi(u)$ is $C^{1}$.

Now we shall investigate the boundary behaviour of $J_{s, G}$

Lemma 13. Let $\left\{u_{k}=\left(u_{k_{1}}, \cdots, u_{k_{n}}\right)\right\} \subset \Lambda_{s, G} D$ and $u_{k} \rightarrow u$ weakly in $\Lambda_{s, G} D$ with $u=$ $\left(u_{1} \cdots, u_{n}\right) \in \partial \Lambda_{s, G} D$. Then $J\left(u_{k}\right) \rightarrow+\infty$.

Proof. Since $\int_{a}^{b} \int_{a}^{b}\left[\sum_{i=1}^{n} G\left(\frac{\left|u_{k_{i}}(x)-u_{k_{i}}(y)\right|}{|x-y|^{s}}\right)\right] \frac{d x d y}{|x-y|}+\int_{a}^{b} \alpha(x)\left|u_{k}(x)\right|^{p(x)} d x>0$, it suffices to show that

$$
\int_{a}^{b} \frac{m(x)}{\left|u_{k}(x)\right|^{\gamma(x)}} d x \rightarrow+\infty
$$

Since $\frac{m(x)}{\left|u_{k}(x)\right| \gamma(x)}>0$,

$$
\int_{a_{0}}^{b_{0}} \frac{m(x)}{\left|u_{k}(x)\right|^{\gamma(x)}} d x \rightarrow+\infty .
$$

for some subinterval $\left(a_{0}, b_{0}\right)$ of $(a, b)$. Since $u \in \partial \Lambda_{s, G} D$, there exists $x_{0} \in(a, b)$ and a neighbourhood $N_{\epsilon}\left(x_{0}\right)=\left\{x \in(a, b)|| x-x_{0} \mid<\epsilon\right\}$ of $x_{0}$ such that $u\left(x_{0}\right) \in \partial D$ and for any $x \in N_{\epsilon}\left(x_{0}\right)$,

$$
\frac{m(x)}{|u(x)|^{\gamma(x)}} \geq \frac{c_{1}}{\left|u(x)-u\left(x_{0}\right)\right|}-c_{2}
$$

for some $c_{1}>0$ and $c_{2}>0$. Thus we have

$$
\int_{N_{\epsilon}\left(x_{0}\right)} \frac{m(x)}{|u(x)|^{\gamma(x)}} d x \geq \int_{N_{\epsilon}\left(x_{0}\right)}\left[\frac{c_{1}}{\left|u(x)-u\left(x_{0}\right)\right|}-c_{2}\right] d x
$$


$\forall \epsilon>0$. Thus

$$
\begin{aligned}
& \left|u(x)-u\left(x_{0}\right)\right| \leq\|u(x)\|_{W^{s} L_{G}\left(S^{1}, R^{n}\right)} \frac{\left|x-x_{0}\right|}{b-a} \\
& \left.\leq \frac{\left|x-x_{0}\right|}{b-a}\left(\int_{a}^{b} \int_{a}^{b}\left[\sum_{i=1}^{n} G\left(\frac{\left|u_{i}(x)-u_{i}(y)\right|}{|x-y|^{s}}\right)\right] \frac{d x d y}{|x-y|}\right]\right)^{\frac{1}{g_{0}}} \\
& \left.<\epsilon\left(\int_{a}^{b} \int_{a}^{b}\left[\sum_{i=1}^{n} G\left(\frac{\left|u_{i}(x)-u_{i}(y)\right|}{|x-y|^{s}}\right)\right] \frac{d x d y}{|x-y|}\right]\right)^{\frac{1}{g_{0}}} .
\end{aligned}
$$

From (17) and (18),

$$
\begin{aligned}
& \int_{N_{\epsilon}\left(x_{0}\right)} \frac{m(x)}{|u(x)| \gamma(x)} d x \\
& \geq \int_{N_{\epsilon}\left(x_{0}\right)}\left[\frac{c_{1}}{\left.\epsilon\left(\int_{a}^{b} \int_{a}^{b}\left[\sum_{i=1}^{n} G\left(\frac{\left|u_{i}(x)-u_{i}(y)\right|}{|x-y|^{s}}\right)\right] \frac{d x d y}{|x-y|}\right]\right)^{\frac{1}{\delta_{0}}}}-c_{2}\right] d x .
\end{aligned}
$$

Then we have

$$
\int_{N_{\epsilon}\left(x_{0}\right)} \frac{m(x)}{|u(x)|^{\gamma(x)}} d x \rightarrow \infty
$$

Now, we shall prove that $J_{s, G}(u)$ satisfies $(P . S.) \tau_{s, G}$ condition for any $\tau_{s, G}>\tau_{s, G}{ }^{0}$ in $\Lambda_{s, G} D$.

Lemma 14. Let $\left(u_{k}\right)_{k}$ be a sequence such that $J_{s, G}\left(u_{k}\right) \rightarrow \tau_{s, G}$ and $D J_{s, G}\left(u_{k}\right) \rightarrow \theta$ for some $\tau_{s, G}>0$. Then if $\left\|u_{k}\right\|_{H} \rightarrow \infty$, then $J_{s, G}\left(u_{k}\right) \leq \tau_{s, G}{ }^{0}$ in $\Lambda_{s, G} D$ for some constant $\tau_{s, G}{ }^{0}>0$.

Proof. Let $u_{k}=\left(u_{k_{1}}, \cdots, u_{k_{n}}\right) \in \Lambda_{s, G} D \subset H$. Assume that $\left\|u_{k}\right\|_{H} \rightarrow \infty$. Since $D J_{s, G}\left(u_{k}\right)=$ $(-\Delta)_{g}^{s} u_{k}+\operatorname{grad}_{u}\left(\alpha(x)\left|u_{k}(x)\right|^{p(x)}\right)+\operatorname{grad}_{u}\left(m(x) \frac{1}{\left|u_{k}\right| \gamma(x)}\right) \rightarrow \theta$, we have, for $u_{k} \in \Lambda_{s, G} D$,

$$
\begin{aligned}
\|u\|_{H} \geq & D J_{s, G}\left(u_{k}\right) \cdot u_{k} \\
= & \int_{a}^{b}(-\Delta)_{g}^{s} u_{k} \cdot u_{k}(x) d x+\int_{a}^{b}\left(\operatorname{grad}_{u}\left(\alpha(x)\left|u_{k}(x)\right|^{p(x)}\right)\right) \cdot u_{k}(x) d x \\
& \quad+\int_{a}^{b}\left(\operatorname{grad}_{u}\left(m(x) \frac{1}{\left|u_{k}\right| \gamma(x)}\right)\right) \cdot u_{k}(x) d x .
\end{aligned}
$$

By (6), we have

$$
\begin{aligned}
& D J_{s, G}\left(u_{k}\right) \cdot u_{k} \\
& =\int_{a}^{b} \int_{a}^{b}\left[\sum_{i=1}^{n} g\left(\frac{\left|u_{k_{i}}(x)-u_{k_{i}}(y)\right|}{|x-y|^{s}}\right) \frac{u_{k_{i}}(x)-u_{k_{i}}(y)}{\left|u_{k_{i}}(x)-u_{k_{i}}(y)\right|} \frac{u_{k_{i}}(x)-u_{k_{i}}(y)}{|x-y|^{s}}\right] \frac{d x d y}{|x-y|} \\
& \quad+\int_{a}^{b}\left[\left(\operatorname{grad}_{u}\left(\alpha(x)\left|u_{k}(x)\right|^{p(x)}\right)\right) \cdot u_{k}(x)+\left(\operatorname{grad}_{u}\left(m(x) \frac{1}{\left|u_{k}\right|^{\gamma(x)}}\right)\right) \cdot u_{k}(x)\right] d x \\
& \geq g_{0}\left[\int_{a}^{b} \int_{a}^{b}\left[\sum_{i=1}^{n} G\left(\frac{\left|u_{k_{i}}(x)-u_{k_{i}}(y)\right|}{|x-y|^{s}}\right)\right] \frac{d x d y}{|x-y|}\right. \\
& +\int_{a}^{b}\left(\operatorname{grad}_{u}\left(\alpha(x)\left|u_{k}(x)\right|^{p(x)}\right)\right) \cdot u_{k}(x) d x+\int_{a}^{b}\left(\operatorname{grad}_{u}\left(m(x) \frac{1}{\left|u_{k}\right|^{\gamma(x)}}\right)\right) \cdot u_{k}(x) d x .
\end{aligned}
$$

for large $k$. By Lemma 7, (20) becomes that 


$$
\begin{aligned}
\left\|u_{k}\right\|_{H} \geq & D J_{s, G}\left(u_{k}\right) \cdot u_{k} \\
\geq & g_{0}\left[\int_{a}^{b} \int_{a}^{b}\left[\sum_{i=1}^{n} G\left(\frac{\left|u_{k_{i}}(x)-u_{k_{i}}(y)\right|}{|x-y|^{s}}\right)\right] \frac{d x d y}{|x-y|}\right. \\
& \quad+\int_{a}^{b}\left(\operatorname{grad}_{u}\left(\alpha(x)\left|u_{k}(x)\right|^{p(x)}\right)\right) \cdot u_{k}(x) d x+\int_{a}^{b}\left(\operatorname{grad}_{u}\left(m(x) \frac{1}{\left|u_{k}\right| \gamma(x)}\right)\right) \cdot u_{k}(x) d x \\
\geq & g_{0}\left\|u_{k}\right\|_{H}^{g_{0}}+\int_{a}^{b}\left(\operatorname{grad}_{u}\left(\alpha(x)\left|u_{k}(x)\right|^{p(x)}\right)\right) \cdot u_{k}(x) d x \\
\quad & \quad \int_{a}^{b}\left(\operatorname{grad}_{u}\left(m(x) \frac{1}{\left|u_{k}\right|^{\gamma(x)}}\right)\right) \cdot u_{k}(x) d x
\end{aligned}
$$

and

$$
\left\|u_{k}\right\|_{H} \geq g_{0}\left\|u_{k}\right\|_{H}^{g_{0}}+\int_{a}^{b}\left(\operatorname{grad}_{u}\left(\alpha(x)\left|u_{k}(x)\right|^{p(x)}\right)\right) \cdot u_{k}(x) d x+\int_{a}^{b}\left(\operatorname{grad}_{u}\left(m(x) \frac{1}{\left|u_{k}\right| \gamma(x)}\right)\right) \cdot u_{k}(x) d x .
$$

By (22) and Hölder inequality in $\Lambda_{s, G} D \subset W^{s} L_{G}\left(S^{1}, R^{n}\right)$, we have

$$
\begin{aligned}
& g_{0}\left\|u_{k}\right\|_{H}^{g_{0}}-\left\|u_{k}\right\|_{H} \\
\leq & -\int_{a}^{b}\left(\operatorname{grad}_{u}\left(\alpha(x)\left|u_{k}(x)\right|^{p(x)}\right)\right) \cdot u_{k}(x) d x-\int_{a}^{b}\left(\operatorname{grad}_{u}\left(m(x) \frac{1}{\left|u_{k}\right| \gamma(x)}\right)\right) \cdot u_{k}(x) d x \\
\leq & 2\left\|\operatorname{grad}_{u}\left(\alpha(x)\left|u_{k}(x)\right|^{p(x)}\right)\right\|_{L_{G^{*}}}\left\|u_{k}\right\|_{L_{G}}+2\left\|\operatorname{grad}_{u}\left(m(x) \frac{1}{\left|u_{k}\right| \gamma(x)}\right)\right\|_{L_{G^{*}}}\left\|u_{k}\right\|_{L_{G}} \\
\leq & 2\left\|\operatorname{grad}_{u}\left(\alpha(x)\left|u_{k}(x)\right|^{p(x)}\right)\right\|_{L_{G^{*}}}\left\|u_{k}\right\|_{H}+2\left\|\operatorname{grad}_{u}\left(m(x) \frac{1}{\left|u_{k}\right| \gamma(x)}\right)\right\|_{L_{G^{*}}}\left\|u_{k}\right\|_{H} .
\end{aligned}
$$

From (23),

$$
g_{0}\left\|u_{k}\right\|_{H}^{g_{0}-1} \leq 1+2\left\|\operatorname{grad}_{u}\left(\alpha(x)\left|u_{k}(x)\right|^{p(x)}\right)\right\|_{L_{G^{*}}}+2\left\|\operatorname{grad}_{u}\left(m(x) \frac{1}{\left|u_{k}\right| \gamma(x)}\right)\right\|_{L_{G^{*}}} .
$$

$\left\|u_{k}\right\|_{H} \rightarrow \infty$ implies that there exists a constant $R_{0}>0$ such that $\left\|u_{k}\right\|_{H} \geq R_{0}$ for large $k$. Thus we have

$$
\int_{a}^{b} \frac{m(x)}{\left|u_{k}\right| \gamma(x)} \leq(b-a)|m(x)|_{\infty} \sup \left\{\frac{1}{\left|u_{k}\right| \gamma(x)} \mid x \in(a, b), u_{k} \in H \backslash B_{R_{0}}\right\} .
$$

By Lemma 9, the embedding

$$
W^{s} L_{G}\left(S^{1}, R^{n}\right) \hookrightarrow L^{r(x)}\left(S^{1}, R^{n}\right)
$$

is continuous and compact for all $1 \leq r(x)<g_{0}^{*}$. Since $p(x)<g_{0}^{*}$, there exists a positive constant $C$ such that

$$
\left\|u_{k}\right\|_{L^{p(x)}} \leq C\left\|u_{k}\right\|_{H}
$$

By (24)-(26), we have 


$$
\begin{aligned}
J_{s, G}\left(u_{k}\right)= & \int_{a}^{b} \int_{a}^{b}\left[\sum_{i=1}^{n} G\left(\frac{\left|u_{k_{i}}(x)-u_{k_{i}}(y)\right|}{|x-y|^{S}}\right)\right] \frac{d x d y}{|x-y|} \\
& +\int_{a}^{b} \alpha(x)\left|u_{k}(x)\right|^{p(x)} d x+\int_{a}^{b} m(x) \frac{1}{\left|u_{k}\right| \gamma(x)} d x \\
\leq & \left\|u_{k}\right\|^{g^{0}}+|\alpha(x)|_{\infty}\left\|u_{k}(x)\right\|_{L^{p(x)}(a, b), R^{n}}^{p(x)}+(b-a)|m(x)|_{\infty} \sup \left\{\frac{1}{\left|u_{k}(x)\right|^{\gamma(x)}}\right\} \\
\leq & \left\|u_{k}\right\|^{g^{0}}+C|\alpha(x)|_{\infty}\left\|u_{k}(x)\right\|_{H}^{p(x)}+(b-a)|m(x)|_{\infty} \sup \left\{\frac{1}{\left|u_{k}(x)\right| \gamma(x)}\right\} \\
\leq & {\left[\frac{1}{g_{0}}\left(1+2\left\|\operatorname{grad}_{u}\left(\alpha(x)\left|u_{k}(x)\right|^{p(x)}\right)\right\| L_{G^{*}}+2 \| \operatorname{grad}_{u}\left(m(x) \frac{1}{\left|u_{k}\right| \gamma(x)} \|_{L_{G^{*}}}\right)\right]^{\frac{g^{0}}{g_{0}-1}}\right.} \\
& +C|\alpha(x)|_{\infty}\left(\left[\frac { 1 } { g _ { 0 } } \left(1+2\left\|\operatorname{grad}_{u}\left(\alpha(x)\left|u_{k}(x)\right|^{p(x)}\right)\right\|_{L_{G^{*}}}\right.\right.\right. \\
& \left.\left.+2 \| \operatorname{grad}_{u}\left(m(x) \frac{1}{\left|u_{k}\right| \gamma(x)} \|_{L_{G^{*}}}\right)\right]^{\frac{p_{+}}{g_{0}-1}}\right)+(b-a)|m(x)|_{\infty} \sup \left\{\frac{1}{\left|u_{k}(x)\right|^{\gamma(x)}}\right\} .
\end{aligned}
$$

If we set,

$$
\begin{aligned}
\tau_{s, G}{ }^{0}=\left[\frac{1}{g_{0}}\right. & \left(1+2\left\|\operatorname{grad}_{u}\left(\alpha(x)\left|u_{k}(x)\right|^{p(x)}\right)\right\|_{L_{G^{*}}}+2\left\|\operatorname{grad}_{u}\left(m(x) \frac{1}{\left|u_{k}\right| \gamma(x)}\right)\right\|_{L_{G^{*}}}\right]^{\frac{g^{0}}{g_{0}-1}} \\
& +C|\alpha(x)|_{\infty}\left(\left[\frac { 1 } { g _ { 0 } } \left(1+2\left\|\operatorname{grad}_{u}\left(\alpha(x)\left|u_{k}(x)\right|^{p(x)}\right)\right\|_{L_{G^{*}}}\right.\right.\right. \\
& \left.\left.+2\left\|\operatorname{grad}_{u}\left(m(x) \frac{1}{\left|u_{k}\right|^{\gamma(x)}}\right)\right\|_{L_{G^{*}}}\right]^{\frac{p_{+}}{g_{0}-1}}\right)+(b-a)|m(x)|_{\infty} \sup \left\{\frac{1}{\left|u_{k}(x)\right| \gamma(x)}\right\},
\end{aligned}
$$

we have $J_{s, G}\left(u_{k}\right) \leq \tau_{s, G}{ }^{0}$.

Lemma 15. $J_{s, G}(u)$ satisfies the $(P . S.) \tau_{s, G}$ condition for any $\tau_{s, G}>\tau_{s, G}{ }^{0}$ in $\Lambda_{s, G} D$.

Proof. Let $\tau_{s, G}>0$ and $\left(u_{k}\right)_{k} \subset \Lambda_{s, G} D$ be a sequence such that $J_{s, G}\left(u_{k}\right) \rightarrow \tau_{s, G}$ and $D J_{s, G}\left(u_{k}\right) \rightarrow \theta, \theta=(0, \cdots, 0)$ in $\Lambda_{s, G} D$. First we shall show that $\left\{u_{k}\right\}$ is bounded in $\Lambda_{s, G} D$. Suppose that $\left\|u_{k}\right\|_{H} \rightarrow \infty$ as $k \rightarrow \infty$. By Lemma 14, $J_{s, G}\left(u_{k}\right) \leq \tau_{s, G}{ }^{0}$, where $\tau_{s, G}{ }^{0}$ is introduced in the proof of Lemma 14. This leads to a contradiction because $J_{s, G}\left(u_{k}\right) \rightarrow$ $\tau_{s, G}>\tau_{s, G}{ }^{0}$. Thus $\left\{u_{k}\right\}$ is bounded in $\Lambda_{s, G} D$. Up to a subsequence, $\left\{u_{k}\right\}$ converges weakly to some $u \in \Lambda_{s, G} D$. We claim that $\left\{u_{k}\right\}$ converges strongly to $u \in \Lambda_{s, G} D$. Since $D J_{s, G}\left(u_{k}\right) \rightarrow \theta$, we have

$$
u_{k}(x)+\left((-\Delta)_{g}^{S}\right)^{-1}\left(\operatorname{grad}_{u}\left(\alpha(x)\left|u_{k}(x)\right|^{p(x)}\right)+\operatorname{grad}_{u}\left(m(x) \frac{1}{\left|u_{k}\right| \gamma(x)}\right)\right) \rightarrow \theta
$$

and we also have

$$
\begin{gathered}
D J_{s, G}\left(u_{k}(x)\right) \cdot u_{k}(x)=(-\Delta)_{g}^{S} u_{k}(x) \cdot u_{k}(x)+\left(\operatorname{grad}_{u}\left(\alpha(x)\left|u_{k}(x)\right|^{p(x)}\right)\right) \cdot u_{k}(x) \\
+\left(\operatorname{grad}_{u}\left(m(x) \frac{1}{\left|u_{k}\right| \gamma(x)}\right)\right) \cdot u_{k}(x) \longrightarrow 0
\end{gathered}
$$

The second and third part of the right-hand side of (28) satisfy

$$
\int_{a}^{b}\left[\operatorname{grad}_{u}\left(\alpha(x)\left|u_{k}(x)\right|^{p(x)} \cdot u_{k}(x)\right] d x \leq p_{+}|\alpha(x)|_{\infty}\left\|u_{k}(x)\right\|_{L^{p(x)}\left(S^{1}, R^{n}\right)}^{p_{+}}\right.
$$


and

$$
\int_{a}^{b}\left(\operatorname{grad}_{u}\left(m(x) \frac{1}{\left|u_{k}\right|^{\gamma(x)}}\right) \cdot u_{k} d x \leq|m(x)|_{\infty} \gamma_{+} \int_{a}^{b} \frac{1}{\left|u_{k}(x)\right|^{\gamma(x)}} d x .\right.
$$

Continuous and compact embedding $W^{s} L_{G}\left(S^{1}, R^{n}\right) \hookrightarrow L^{p(x)}\left(S^{1}, R^{n}\right)$ for $1<p(x)<$ $g_{0}^{*}$ implies that $\left\|u_{k}\right\|_{L^{p(x)}\left(S^{1}, R^{n}\right)} \leq C\left\|u_{k}\right\|_{H}$ for some constant $C>0$. Thus if $\left\{u_{k}\right\}$ is bounded in $\Lambda_{s, G} D \subset W^{s} L_{G}\left(S^{1}, R^{n}\right)$, then $\left\{u_{k}\right\}$ is bounded in $L^{p(x)}\left(S^{1}, R^{n}\right)$. It follows that $\int_{a}^{b}\left[\operatorname{grad}_{u}\left(\alpha(x)\left|u_{k}(x)\right|^{p(x)} \cdot u_{k}(x)\right] d x\right.$ and $\int_{a}^{b}\left(\operatorname{grad}_{u}\left(m(x) \frac{1}{\left|u_{k}\right| \gamma(x)}\right) \cdot u_{k} d x\right.$ are bounded in $L^{p(x)}\left(S^{1}, R^{n}\right)$. By $(28),(-\Delta)_{g}^{s} u_{k}(x) \cdot u_{k}(x)$ is bounded. Since $\left\{u_{k}\right\}$ is bounded in $\Lambda_{s, G} D$,

$$
(-\Delta)_{g}^{s} u_{k}(x) \text { is bounded in } L^{p(x)}\left(S^{1}, R^{n}\right) .
$$

Lemma 9 leads that $\left\{u_{k}\right\}$ converges to $u$ strongly in $\Lambda_{s, G} D$.

\section{Proofs of Theorems 1 and 2}

Let us set the level set

$$
J_{s, G} \tau_{s, G}=\left\{u \in \Lambda_{s, G} D \mid J_{s, G}(u) \leq \tau_{s, G}\right\} .
$$

Lemma 16. $H_{b_{i}}\left(\Lambda_{s, G} D\right) \neq 0$ for some strictly increasing sequence $b_{i}$.

Proof. Let $\bar{C}_{\epsilon}(\theta)$ be a neighbourhood $\theta$ with radius $\epsilon>0$ and choose $R>0$ such that $\bar{C}_{\epsilon}(\theta) \subset \operatorname{int}\left(B_{R}(\theta)\right)$. Then we have

$$
R^{n} \backslash B_{R}(\theta) \subset\left(R^{n} \backslash\{\theta\}\right)
$$

and $R^{n} \backslash B_{R}(\theta)$ is a deformation retract of $R^{n} \backslash\{\theta\}$. Then $\Lambda_{s, G}\left(R^{n} \backslash B_{R}(\theta)\right)$ is a deformation retract of $\Lambda_{s, G} D$. Thus we have

$$
\begin{gathered}
H_{*}\left(\Lambda_{s, G} D\right) \cong H_{*}\left(\Lambda_{s, G}\left(R^{n} \backslash B_{R}(\theta)\right)\right) \oplus H_{*}\left(\Lambda_{s, G} D, \Lambda_{s, G}\left(R^{n} \backslash B_{R}(\theta)\right)\right) \\
\cong H_{*}\left(\Lambda_{s, G}\left(S^{n-1}\right) \oplus H_{*}\left(\Lambda_{s, G} D, \Lambda_{s, G}\left(S^{n-1}\right)\right) .\right.
\end{gathered}
$$

In [111], the Poincar series of $\Lambda_{s, G}\left(S^{n-1}\right)$ is written as

$$
P_{t}\left(\Lambda_{s, G}\left(S^{n-1}\right)\right)=\left(1+t^{n}\right)+\frac{t^{n-1}}{1-t^{2(n-1)}}\left(1+t^{n}\right)\left(1+t^{n-1}\right) .
$$

Lemma 17. The level set $J_{s, G} \tau_{s, G}$ is deformed into $\Sigma_{s, G}$ for some finite dimensional singular complex $\Sigma_{s, G}=\Sigma_{\tau_{s, G}}$.

Proof. Let $u \in J_{s, G} \tau_{\tau_{s, G}}$. Then $u=\left(u_{1}, \cdots, u_{n}\right) \in \Lambda_{s, G} D$ and

$$
\begin{aligned}
J_{s, G}(u)=\int_{a}^{b} & \int_{a}^{b}\left[\sum_{i=1}^{n} G\left(\frac{\left|u_{i}(x)-u_{i}(y)\right|}{|x-y|^{s}}\right)\right] \frac{d x d y}{|x-y|} \\
& +\int_{a}^{b} \alpha(x)|u(x)|^{p(x)} d x+\int_{a}^{b} m(x) \frac{1}{|u| \gamma(x)} d x \leq \tau_{s, G} .
\end{aligned}
$$

If $(x, u(x)) \in[a, b] \times\left(R^{n} \backslash B_{R_{0}}(\theta)\right)$, then

$$
0<\frac{m(x)}{|u(x)|^{\gamma(x)}}<+\infty, \text { and }\left|\operatorname{grad}_{u}\left(\frac{m(x)}{|u(x)|^{\gamma(x)}}\right)\right|<+\infty
$$

for some constant $R_{0}>0$. We also note that if $u(x) \in B_{R_{0}} \backslash \bar{C}_{\epsilon}(\theta)$, then 


$$
(b-a) \inf \{\alpha(x)\} \epsilon^{p_{-}}+(b-a) \frac{\inf \{m(x)\}}{R_{0}^{\gamma+}}<\int_{a}^{b} \alpha(x)|u(x)|^{p(x)} d x+\int_{a}^{b} \frac{m(x)}{|u(x)|^{\gamma(x)}} d x<\tau_{s, G} .
$$

(29)-(31) imply that

$$
\int_{a}^{b} \int_{a}^{b}\left[\sum_{i=1}^{n} G\left(\frac{\left|u_{i}(x)-u_{i}(y)\right|}{|x-y|^{s}}\right] \frac{d x d y}{|x-y|}<\tau_{s, G}{ }^{1}\right.
$$

for some constant $\tau_{s, G}{ }^{1}>0$. By Lemma 13 , there exists $\epsilon_{s, G}{ }^{0}=\epsilon_{s, G}\left(\tau_{s, G}, \tau_{s, G}{ }^{1}\right)$ such that

$$
d\left(u, \bar{C}_{\epsilon_{s, G}}(\theta)\right) \geq \epsilon_{s, G}{ }^{0} \quad \forall u \in J_{s, G} \tau_{\tau_{s, G}}, \forall x \in[a, b] .
$$

Let us choose an integer $N_{s, G}=N_{\tau_{s, G}}>\frac{\left(\tau_{s, G}\right)^{\frac{1}{g_{0}}}}{\epsilon_{s, G^{0}}}$ and set

$$
x_{s, G}{ }^{i}=\frac{(b-a) i}{N_{s, G}} \quad i=1,2, \cdots, N_{s, G}
$$

and

$$
\bar{u}_{s, G}(x)=\left(1-\frac{1}{b-a} N_{s, G}\left(x-x_{s, G}^{i-1}\right)\right) u\left(x_{s, G}^{i-1}\right)+\frac{1}{b-a} N_{s, G}\left(x-x_{s . G}^{i-1}\right) u\left(x_{s, G}{ }^{i}\right)
$$

$\forall x \in\left[x_{s, G}{ }^{i-1}, x_{s, G}{ }^{i}\right], i=1,2, \ldots, N_{s, G}, \forall u \in J_{s, G} \tau_{s, G}$. Let

$$
\Sigma_{s, G}=\left\{\bar{u}_{s, G}(x) \mid u \in J_{s, G} \tau_{s, G}\right\}
$$

Mapping $D \times D \times \cdots \times{ }_{N_{s, G}} D \ni \bar{u}_{s, G} \mapsto\left(u\left(x_{s, G}^{1}\right), u\left(x_{s, G}{ }^{2}\right), \ldots, u\left(x_{s, G} N_{s, G}\right)\right)$ is a homeomorphism. We shall show that $\Sigma_{s, G} \subset \Lambda_{s, G} D$. In fact, for $\forall u \in J_{s, G} \tau_{s, G}$ and $x_{s, G}{ }^{i}>x_{s, G}{ }^{i-1}$, by (32), we have

$$
\begin{aligned}
& \left|u\left(x_{s, G}{ }^{i}\right)-u\left(x_{s, G}{ }^{i-1}\right)\right| \leq\left\|\bar{u}_{s, G}(x)\right\|_{W^{s} L_{G}\left(S^{1}, R^{n}\right)} \frac{\left|x_{s, G}{ }^{i}-x_{s, G}{ }^{i-1}\right|}{b-a} \\
& \left.\leq \frac{\left|x_{s, G}{ }^{i}-x_{s, G}{ }^{i-1}\right|}{b-a}\left(\int_{a}^{b} \int_{a}^{b}\left[\sum_{i=1}^{n} G\left(\frac{\left|u_{i}(x)-u_{i}(y)\right|}{|x-y|^{s}}\right)\right] \frac{d x d y}{|x-y|}\right]\right)^{\frac{1}{g_{0}}} \\
& <\frac{\epsilon_{s, G}}{b-a}\left(\tau_{s, G}{ }^{1}\right)^{\frac{1}{g_{0}}}
\end{aligned}
$$

Thus

$$
\begin{aligned}
d\left(\bar{u}_{s, G}(x), \bar{C}_{\epsilon_{s, G}}(\theta)\right) \geq & d\left(u\left(x_{s, G}{ }^{i}\right), \bar{C}_{\epsilon_{s, G}}(\theta)\right) \\
& \quad-\left(1-\frac{1}{b-a} N_{s, G}\left(x-x_{s, G}{ }^{i-1}\right)\right)\left|u\left(x_{s, G}{ }^{i}\right)-u\left(x_{s, G}{ }^{i-1}\right)\right| \\
\geq & \epsilon_{s, G}{ }^{0}-\left(N_{s, G}\right)^{-1}\left(\tau_{s, G}{ }^{1}\right)^{\frac{1}{g_{0}}}>0
\end{aligned}
$$

$\forall x \in\left[x_{s, G}{ }^{i-1}, x_{s, G}{ }^{i}\right]$. We shall show that there exists $\eta_{s, G} \in C\left([0,1] \times J_{s, G} \tau_{s, G}, \Lambda_{s, G} D\right)$ such that $\eta_{s, G}(0, \cdot)=\mathrm{id}$, and $\eta_{s, G}\left(1, J_{s, G} \tau_{s, G}\right)=\Sigma_{s, G}$. In fact, Let us choose $u(x) \in \Lambda_{s, G} D$ and let us define $\eta_{s, G}$ as follows:

$$
\eta_{s, G}(y, u)(x)=\left\{\begin{array}{lc}
u(x) & \text { for } x \geq(b-a) y, \\
\left(1-\frac{x-x_{s, G}{ }^{i-1}}{(b-a) y-x_{s, G}{ }^{i-1}}\right) u\left(x_{s, G}{ }^{i-1}\right)+\frac{x-x_{s, G}{ }^{i-1}}{(b-a) y-x_{s, G}} u((b-a) y) & \text { for } x_{s, G}{ }^{i-1}<x<(b-a) y, \\
\bar{u}_{s, G}(x) & \text { for } x \leq x_{s, G}{ }^{i-1} .
\end{array}\right.
$$


Then $\eta_{s, G}(0, \cdot)=\mathrm{id}$, and $\eta_{s, G}\left(1, J_{s, G} \tau_{\tau_{s, G}}\right)=\Sigma_{s, G}$. Thus we prove that $J_{s, G}$ is deformed into $\Sigma_{s, G}$ in the loop space $\Lambda_{s, G} D$.

Proof of Theorem 1. We claim that for each $q>n N_{s, G}$, where $N_{s, G}=N_{s, G} \tau_{s, G}$ is defined in Lemma 17, let us set

$$
\tau_{s, G}=\inf _{z \in \beta} \max _{u \in[z]} J_{s, G}(u),
$$

where $\beta \in H_{q}\left(\Lambda_{s, G} D\right)$ is nontrivial. We claim that $\tau_{s, G}>\tau_{s, G}{ }^{0}$ and $\tau_{s, G}$ is a critical value of $J_{s, G}$. In fact, suppose that $\tau_{s, G} \leq \tau_{s, G}{ }^{0}$ and $\tau_{s, G}$ is not a critical value of $J_{s, G}$. Then $[u] \in J_{s, G} \tau_{\tau_{s, G}{ }^{0}+1}$ for some $[u] \in \beta$. By Lemma 17, there exists a deformation $\eta_{s, G}:[0,1] \times$

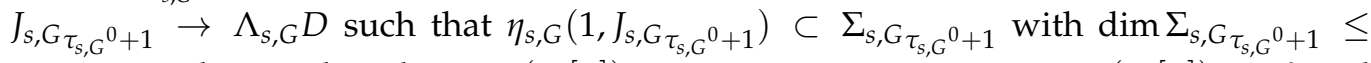
$n N_{s, G} \tau_{\tau_{s, G}}$. This implies that $\eta_{s, G}(1,[u]) \subset \Sigma_{s, G} \tau_{\tau_{s, G}{ }^{0}+1}$. However, $\eta_{s, G}(1,[u]) \in \beta$ and $\beta \in H_{q}\left(\Lambda_{s, G} D\right)$ with $q>n N_{s, G} \tau_{\tau_{s, G}}$, which is absurd.

Proof of Theorem 2. We suppose that $J_{s, G}(u)$ has only finitely many critical points $u_{1}, u_{2}$, $\ldots, u_{m_{s, G}}$ such that we can obtain $J_{s, G}\left(u_{k}\right)>\tau_{s, G}{ }^{0}, 1 \leq k \leq m_{s, G}$. Let us set

$$
K=\left\{u_{1}, u_{2}, \ldots, u_{m_{s, G}}\right\} .
$$

We note that $\operatorname{dim} \operatorname{ker}\left(D^{2} J_{s, G}\left(u_{k}\right)\right) \leq 2 n$, for all $k$. Letting

$$
b_{s, G}^{*}>\max \left\{n N_{s, G} \tau_{s, G}, \operatorname{ind}\left(J_{s, G}, u_{k}\right)+\operatorname{dim} \operatorname{ker}\left(D^{2} J_{s, G}\left(u_{k}\right)\right) \mid 1 \leq k \leq m_{s, G}\right\} .
$$

and

$$
d_{s, G}>\max \left\{\tau_{s, G}^{0}, J_{s, G}\left(u_{k}\right) \mid 1 \leq k \leq m_{s}, G\right\},
$$

we have

$$
C_{b}\left(J_{s, G}, u_{k}\right)=0 \quad \forall b_{s, G} \geq b_{s, G}^{*}, k=1,2, \ldots, m_{s, G}
$$

and

$$
H_{*}\left(\Lambda_{s, G} D, J_{s, G} \tau_{\tau_{s, G} 0}\right)=H_{*}\left(J_{s, G_{d_{s, G}}} J_{s, G_{\tau_{s, G}}}\right) .
$$

Hence

$$
H_{b}\left(\Lambda_{s, G} D, J_{s, G} \tau_{s, G}\right)=0 \quad \forall b_{s, G}>b_{s, G}^{*}
$$

Since

$$
i_{*}: H_{b}\left(\Lambda_{s, G} D\right) \longrightarrow H_{b}\left(\Lambda_{s, G} D, J_{s, G} \tau_{s, G}\right) \text { is injective for } b_{s, G} \geq b_{s, G}^{*}
$$

we have

$$
H_{b}\left(\Lambda_{s, G} D\right)=0 \text { for } b_{s, G} \geq b_{s, G}^{*}
$$

which is absurd.

Author Contributions: Q.-H.C. introduced the main ideas of multiplicity study for this problem. T.J. participate in applying the method for solving this problem and drafted the manuscript. All authors contributed equally to read and approved the final manuscript.

Funding: Q.-H.C. was supported by Basic Science Research Program through the National Research Foundation of Korea(NRF) funded by the Ministry of Education, Science and Technology (NRF2017R1D1A1B03030024). T.J. was supported by Basic Science Research Program through the National Research Foundation of Korea(NRF) funded by the Ministry of Science, ICT and Future Planning (NRF-2017R1A2B4005883).

Institutional Review Board Statement: Not applicable.

Informed Consent Statement: Not applicable.

Data Availability Statement: Not applicable.

Acknowledgments: Not applicable. 
Conflicts of Interest: The authors declare no conflict of interest.

\section{References}

1. Applebaum, D. Lévy processes-From Probability to Finance and Quantum Groups, Notices. AMS 2004, 51, $1336-1347$.

2. Chen, Y.; Levine, S.; Rao, M. Variable exponent, linear growth functionals in image restoration. SIAM J. Appl. Math. 2006, 66, 1383-1406. [CrossRef]

3. Cont, R.; Tankov, P. Financial Modelling with Jump Processes; Financial Mathematics Series; Chapman and Hall/CRC: Boca Raton, FL, USA, 2004; Volume 2.

4. Gossez, J.P. Nonlinear elliptic boundary value problems for equations with rapidly (or slowly) increasing coefficients. Trans. Am. Math. Soc. 1974, 190, 163-205. [CrossRef]

5. Hsini, M.; Irzi, N.; Kefi, K. On a fractional problem with variable exponent. Proc. Rom. Acad. Ser. Math. Phys. Tech. Sci. Inf. Sci. 2019, 71, 223-237.

6. Mihailescu, M.; Radulescu, V. Neumann problems associated to nonhomogeneous differential operators in Orlicz-Sobolev spaces Ann. Inst. Fourier 2008, 58, 2087-2111. [CrossRef]

7. Musielak, J. Orlicz Spaces and Modular Spaces; Springer: Berlin, Germany, 1983.

8. Pezzo, L.M.; Rossi, J.D. Trace for fractional Sobolev spaces with variables exponents. arXiv 2017, arXiv:1704.02599.

9. Rao, M.M.; Ren, Z.D. Theory of Orlicz Spaces; Marcel Dekker, Inc.: New York, NY, USA, 1991.

10. Ruzicka, M. Electrorheological Fluids: Modeling and mathematical theory. In Lecture Notes in Mathematics; Springer: Berlin, Germany, 2002; Volume 1748.

11. Samorodnitsky, G.; Taqqu, M.S. Stable Non-Gaussian Random Processes: Stochastic Models with Infinite Variance; Chapman and Hall: New York, NY, USA, 1994; Volume 2.

12. Zhang, C.; Zhang, X. Renormalized solutions for the fractional $p(x)$-Laplacian equation with $L^{1}$ data. arXiv 2017, arXiv:1708.04481v1.

13. Zhang, Q. Existence of solutions for $p(x)$-Laplacian equations with singular coefficients in $R^{N}$. Math. Anal. Appl. 2008, 348, 38-50. [CrossRef]

14. Zhikov, V.V. Averaging of functionals of the calculus of variations and elasticity theory. SSSR Ser. Mat. 1986, 50, 675-710. [CrossRef]

15. Bandle, C. Asymptotic behavior of large solutions of quasilinear elliptic problems. Z. Angew. Math. Phys. 2003, 54, 731-738. [CrossRef]

16. Bandle, C.; Essen, M. On the solutions of quasilinear elliptic problems with boundary blow-up, in partial differential equations of elliptic type (Cortona, 1992). Sympos. Math. 1994, 35, 93-111.

17. Bandle, C.; Marcus, M. 'Large' solutions of semilinear elliptic equations: Existence, uniqueness, and asymptotic behavior. J. Anal. Math. 1992, 58, 9-24. [CrossRef]

18. Bandle, C.; Marcus, M. Dependence of blowup rate of large solutions of semilinear elliptic equations on the curvature of the boundary. Complex Var. Theory Appl. 2004, 49, 555-570. [CrossRef]

19. Du, Y.; Huang, Q. Blow-up solutions for a class of semilinear elliptic and parabolic equations. SIAM J. Math. Anal. 1999, 31, 1-18. [CrossRef]

20. Dunninger, D.R.; Wang, H. It Multiplicity of positive radial solutions for an elliptic system on an annlus domain. Nonlinear Anal. TMA 2000, 42, 5. [CrossRef]

21. Dupaigne, L.; Ghergu, M.; Radulescu, V. Lane-Emden-Fowler equations with convection and singular potential. J. Math. Pures Appl. 2007, 87, 563-581. [CrossRef]

22. Fulks, W.; Maybee, J.S. A singular nonlinear equation. Osaka Math. J. 1960, 12, 1-19.

23. Garcia-Melián, J.; Letelier-Albornoz, R.; de Lis, J.S. Uniqueness and asymptotic behavior for solutions of semilinear problems with boundary blow-up. Proc. Am. Math. Soc. 2001, 129, 3593-3602. [CrossRef]

24. Ghergu, M.; Rádulescu, V. Singular Elliptic Problems, Bifurcation and Asymptotic Analysis; Oxford Lecture Series in Mathematics and Its Applications; Oxford University Press: Oxford, UK, 2008; Volume 37.

25. Lazer, A.C.; McKenna, P.J. Asymptotic behavior of solutions of boundary blowup problems. Differ. Integral Equ. 1994, 7, 1001-1019.

26. Lazer, A.C.; McKenna, P.J. On a problem of Bieberbach and Rademacher. Nonlinear Anal. 1993, 21, 327-335. [CrossRef]

27. Loewner, C.; Nirenberg, L. Partial differential equations invariant under conformal or projective transformations. In Contribution to Analysis; Academic Press: New York, NY, USA, 1974; pp. 245-272.

28. Marcus, M.; Véron, L. Existence and uniqueness results for large solutions of general nonlinear elliptic equations. J. Evol. Equ. 2003, 3, 637-652. [CrossRef]

29. Marcus, M.; Véron, L. Uniqueness and asymptotic behavior of solutions with boundary blow-up for a class of nonlinear elliptic equations. Ann. Inst. Anal. Nonlinéaire 1997, 14, 237-274. [CrossRef]

30. O'Regan, D. Some general existence principles and results for $\left(\phi\left(y^{\prime}\right)\right)^{\prime}=q f\left(t, y, y^{\prime}\right), 0<t<1$. SIAM J. Math. Anal. 1993, 24, 648-668.

31. Rademacher, H. Einige besondere Probleme der partiellen Differentialgleichungen. In Die Differential and Integralgleichungen der Mechanik and Physik I, 2nd ed.; Frank, P., von Mises, R., Eds.; Rosenberg: New York, NY, USA, 1943; pp. $838-845$. 
32. Radulescu, V. Singular phenomena in nonlinear elliptic problems, From blow-up boundary solutions to equations with singular nonlinearities. In Handbook of Differential Equations: Stationary Partial Differential Equations; North-Holland Elsevier Science: Amsterdam, The Netherlands, 2007; Volume 4, pp. 483-591.

33. Ratto, A.; Rigoli, M.; Véron, L. Scalar curvature and conformal deformation of hyperbolic space. J. Funct. Anal. 1994, 121, 15-77. [CrossRef]

34. Shi, J.; Yao, M. On a singular nonlinear semilinear elliptic problem. Proc. R. Soc. Edinburgh. Sect. A 1998, 128, 1389-1401. [CrossRef]

35. Trabelsi, M.; Trabelsi, N. Singular limit solutions for a 2-dimensional semilinear elliptic system of Liouville type. Adv. Nonlinear Anal. 2016, 5, 315-329. [CrossRef]

36. Alves, C.O.; Ferreira, M.C. Existence of solutions for a class of $p(x)$-Laplacian equations involving a concave-convex nonlinearity with critical growth in $R^{N}$. Topol. Methods Nonlinear Anal. 2015, 45, 399-422. [CrossRef]

37. Amrouss, A.R.E.; Kissi, F. Multiplicity of solutions for a general $p(x)$-Laplacian Dirichlet problem. Arab. J. Math. Sci. 2013, 19, 205-216. [CrossRef]

38. Benouhiba, N. Nonlocal eigenvalue problems in variable exponent Sobolev spaces. J. Nonlinear Funct. Anal. 2015, $2015,1-15$.

39. Diening, L. Riesz potential and Sobolev embeddings on generalized Lebesgue and Sobolev spaces $L^{p(\cdot)}$ and $W^{k, p(\cdot)}$. Math. Nachr. 2004, 268, 31-43. [CrossRef]

40. Diening, L.; Harjulehto, P.; Hasto, P.; Ruzicka, M. Lebesgue and Sobolev Spaces with Variable Exponents; Springer: Heidelberg, Germany, 2011.

41. Lê, A. Eigenvalue Problems for the $p$-Laplacian. Nonlinear Anal. 2006, 64, 1057-1099. [CrossRef]

42. Mihailescu, M.; Pucci, P.; Radulescu, V. Eigenvalue problems for anisotropic quasilinear elliptic equations with variable exponent. J. Math. Anal. Appl. 2008, 340, 687-698. [CrossRef]

43. Mihailescu, M.; Radulescu, V. A multiplicity result for a nonlinear degenerate problem arising in the theory of electrorheological fluids. Proc. R. Soc. Lond. Ser. A Math. Phys. Eng. Sci. 2006, 462, 2625-2641. [CrossRef]

44. Rajagopal, K.; Ruzicka, M. Mathematical modeling of electrorheological materials. Contin. Mech. Thermodyn. 2001, 13, 59-78. [CrossRef]

45. Ruiz-Medina, M.D.; Anh, V.V.; Angulo, J.M. Fractional generalized random fields of variable order. Stoch. Anal. Appl. 2004, 22, 775-799. [CrossRef]

46. Zhikov, V.V. On the density of smooth functions in Sobolev-Orlicz spaces. POMI 2004, 226, 67-81. [CrossRef]

47. Amster, P.; Nápoli, P.; Mariani, M.C. Existence of solutions for elliptic systems with critical Sobolev exponent. Electron. J. Differ. Equ. 2002, 2002, 1-13.

48. Bartsch, T.; Guo, Y. Existence and nonexistence results for critical growth polyharmonic elliptic systems. J. Diff. Equ. 2006, 220, 531-543. [CrossRef]

49. Bartsch, T.; Liu, Z. On a superlinear elliptic $p$-Laplacian equation. J. Differ. Equ. 2004, 198, 149-175. [CrossRef]

50. Bartsch, T.; Schneider, M.; Weth, T. Multiple solutions of a critical polyharmonic equation. J. Reine Angew. Math. 2004, 571, 131-143. [CrossRef]

51. Bartsch, T.; Weth, T.; Willem, M. A Sobolev inequality with remainder term and critical equations on domains with topology for the polyharmonic operator. Calc. Var. Partial Differ. Equ. 2003, 18, 253-268. [CrossRef]

52. Boccardo, L.; de Figueiredo, D. Some remarks on a system of quasilinear elliptic equations. Nonlinear Differ. Equ. Appl. NODEA 2002, 9, 309-323. [CrossRef]

53. Brézis, H.; Nirenberg, L. Positive solutions of nonlinear elliptic equations involving critical Sobolev exponents. Comm. Pure Appl. Math. 1983, 36, 437-477. [CrossRef]

54. Dinca, G.; Jebelean, P.; Mawhin, J. Variational and topological methods for Dirichlet problems with $p$-Laplacian. Portugal Math. 2001, 58, 339-378.

55. Drábek, P.; Kufner, A.; Nicolosi, F. Quasilinear Elliptic Equations with Degenerations and Singularities; de Gruyter: Berlin, Germany, 1997.

56. Edmunds, D.E.; Fortunato, D.; Jannelli, E. Critical exponents critical dimensions and biharmonic operators. Arch. Rat. Mech. Anal. 1990, 112, 269-289. [CrossRef]

57. Gazzola, F.; Grunau, H.C.; Squassina, M. Existence and nonexistence results for critical growth biharmonic elliptic equations. Calc. Var. Partial. Differ. Equ. 2003, 18, 117-143. [CrossRef]

58. Grunau, H. Positive solutions to semilinear polyharmonic Dirichlet problems involving critical Sobolev exponents. Calc. Var. 1995, 3, 243-252. [CrossRef]

59. Huang, Y. Existence of positive solutions for a class of the $p$-Laplace equations. J. Austral. Math. Soc. Sect. B 1994, 36, $249-264$. [CrossRef]

60. Kaufmann, U.; Rossi, J.D.; Vidal, R. Fractional Sobolev spaces with variable exponents and fractional $p(x)$-Laplacians. Electron. J. Qual. Theory Differ. Equ. 2017, 76, 1-10. [CrossRef]

61. Montenegro, M. On nontrivial solutions of critical polyharmonic elliptic systems. J. Differ. Equ. 2009, 247, 906-916. [CrossRef]

62. Napoli, P.D.; Mariani, M. Mountain pass solutions to equations of $p$-Laplacin type. Nonlinear Anal. 2003, 54, 1205-1219. [CrossRef]

63. Pucci, P.; Serrin, J. Critical exponents and critical dimensions for polyharmonic operators. J. Math. Pures Appl. $1990,69,55-83$. 
64. Autuori, G.; Pucci, P. Elliptic problems involving the fractional Laplacian in $R^{N}$. J. Differ. Equ. 2013, 255, 2340-2362. [CrossRef]

65. Bahrouni, A. Comparison and sub-supsolution principles for the fractional $p(x)$-Laplacian. J. Math. Anal. Appl. 2018, 458, 1363-1372. [CrossRef]

66. Bahrouni, A.; Radulescu, V. On a new fractional Sobolev space and application to nonlocal variational problems with variable exponent. Discret. Contin. Dyn. Syst. Ser. S 2018, 11, 379-389. [CrossRef]

67. Caffarelli, L.; Silvestre, L. An extension problem related to the frational Laplacian. Commun. Partial Differ. Equ. 2007, 32, 1245-1260. [CrossRef]

68. Farcaseanu, M. On an eigenvalue problem involving the fractional (s,p)-Laplacian. Fract. Calc. Appl. Anal. 2018, 21, 94-103. [CrossRef]

69. Farcaseanu, M.; Mihailescu, M.; Stancu-Dumitru, D. Perturbed fractional eigenvalue problems. Discret. Contin. Dyn. Syst. Ser. A 2017, 37, 6243-6255. [CrossRef]

70. Kikuchi, K.; Negoro, A. On Markov processes generated by pseudodifferential operator of variable order. Osaka J. Math 1997, 34, 319-335.

71. Leopold, H.G. Embedding of function spaces of variable order of differentiation. Czechoslov. Math J. 1999, 49, 633-641. [CrossRef]

72. Lindgren, E.; Lindqvist, P. Fractional eigenvalues. Calc. Var. Partial Differ. Equ. 2014, 49, 795-826. [CrossRef]

73. Lorenzo, C.F.; Hartley, T.T. Initialized fractional calculus. Int. J. Appl. Math. 2000, 3, $249-265$.

74. Lorenzo, C.F.; Hartley, T.T. Variable order and distributed order fractional operators. Nonlinear Dynam. 2002, 29, 57-98. [CrossRef]

75. Mingqi, X.; Bisci, G.M.; Tian, G.; Zhang, B. Infinitely many solutions for the stationary Kirchhoff problems involving the fractional $p$-Laplacian. Nonlinearity 2016, 29, 357-374. [CrossRef]

76. Mingqi, X.; Radulescu, V.D.; Zhang, B. Fractional Kirchhoff problems with critical Trudinger-Moser nonlinearity. Calc. Var. Partial. Differ. Equ. 2019, 58, 57. [CrossRef]

77. Mingqi, X.; Radulescu, V.D.; Zhang, B. Nonlocal Kirchhoff problems with singular exponential nonlinearity. Appl. Math. Optim. 2020. [CrossRef]

78. Bisci, G.M.; Radulescu, V.; Servadei, R. Variational methods for nonlocal fractional problems. In Encyclopedia of Mathematics and Its Applications; Cambridge University Press: Cambridge, UK, 2016; Volume 162.

79. Nezza, E.D.; Palatucci, G.; Valdinoci, E. Hitchhiker's guide to the fractional Sobolev spaces. Bull. Sci. Math. 2012, 136, 521-573. [CrossRef]

80. Pucci, P.; Xiang, M.; Zhang, B. Existence and multiplicity of entire solutions for fractional $p$-Kirchhoff equations. Adv. Nonlinear Anal. 2016, 5, 27-55. [CrossRef]

81. Pucci, P.; Xiang, M.; Zhang, B. Multiple solutions for nonhomogeneous Schrodinger-Kirchhoff type equations involving the fractional $p$-Laplacian in $R^{N}$. Calc. Var. Partial Differ. Equ. 2015, 54, 2785-2806. [CrossRef]

82. Servadei, R.; Valdinoci, E. Mountain pass solutions for non-local elliptic operator. J. Math. Anal. Appl. 2012, 389, 887-898. [CrossRef]

83. Servadei, R.; Valdinoci, E. Variational methods for non-local operators of elliptic type. Discret. Contin. Dyn. Syst. 2013, 33, 2105-2137. [CrossRef]

84. Xiang, M.; Radulescu, V.; Zhang, B. Existence of solutions for perturbed fractional p-Laplacian equations. J. Differ. Equ. 2016, 260, 1392-1413. [CrossRef]

85. Xiang, M.; Wang, F. Fractional Schrodinger-Poisson-Kirchhoff systems involving critical nonlinearities. Nonlineaer Anal. 2017, 164, 1-26. [CrossRef]

86. Xiang, M.; Zhang, B.; Ferrara, M. Existence of solutions for Kirchhoff type problem involving the non-local fractional $p-$ Laplacian. J. Math. Anal. Appl. 2015, 424, 1021-1041. [CrossRef]

87. Xiang, M.; Zhang, B.; Guo, X. Infinitely many solutions for a fractional Kirchhoff type problem via Fountain theorem. Nonlinear Anal. 2015, 120, 299-313. [CrossRef]

88. Bonder, J.F.; Salort, A.M. Fractional order Orlicz-Sobolev spaces. J. Funct. Anal. 2019. [CrossRef]

89. Garciá-Huidobro, M.; Le, V.K.; Manásevich, R.; Schmitt, K. On principle eigenvalues for quasilinear elliptic differential operators: An Orlicz-Sobolev space setting. Nonlineae Differ. Equ. Appl. 1999, 6, 207-225. [CrossRef]

90. Choi, Q.; Jung, T. Existence of solution for $p$-Laplacian boundary value problems with two singular and subcritical nonlinearities. Bound. Value Probl. 2019. [CrossRef]

91. Agawal, R.; Lü, H.; O’Regan, D. A necessary and sufficient condition for the existence of positive solution to the singular p-Laplacian. Z. Anal. Anwend. 2003, 22, 689-709. [CrossRef]

92. Agawal, R.; Lü, H.; O’Regan, D. Existence theorems for the one-dimensional singular $p$-Laplacian equation with sign changing nonlinearities. Appl. Math. Comput. 2003, 143, 15-38.

93. Godoy, P.T.; Kaufmann, U. On Dirichlet problems with singular nonlinearity of indefinite sign. J. Math. Anal. Appl. 2015, 428, 1239-1251. [CrossRef]

94. Kaufmann, U.; Medri, I. One-dimensional singular problems involving the p-Laplacian and nonlinearities indefinite in sign. Adv. Nonlinear Anal. 2016, 5, 251-259. [CrossRef]

95. Kaufmann, U.; Medri, I. Strictly positive solutions for one-dimensional nonlinear problems involving the $p$-Laplacian. Bull. Austral. Math. Soc. 2014, 89, 243-251. [CrossRef] 
96. Kaufmann, U.; Medri, I. Strictly positive solutions for one-dimensional nonlinear elliptic problems. Electron J. Differ. Equ. 2014, $126,13$.

97. Lü, H.; O'Regan, D.; Agawal, R. Positive solutions for singular $p$-Laplacian equations with sign changing nonlinearities using inequality theory. Appl. Math. Comput. 2005, 165, 587-597. [CrossRef]

98. Lü, H.; Zhong, C. A note on singular nonlinear boundary value problems for the one-dimensional p-Laplacian. Appl. Math. Lett. 2001, 14, 189-194. [CrossRef]

99. Ma, D.; Han, J.; Chen, X. Positive solution of three-point boundary value problem for the one-dimensional $p$-Laplacian with singularities. J. Math. Anal. Appl. 2006, 324, 118-133. [CrossRef]

100. Sun, B.; Ge, W. Existence and iteration of positive solutions for some $p$-Laplacian boundary value problems. Nonlinear Anal. 2007, 67, 1820-1830. [CrossRef]

101. Wang, J.; Gao, W. A singular boundary value problem for the one-dimensional p-Laplacian. J. Math. Anal. Appl. 1996, 201, 851-866. [CrossRef]

102. Choi, Q.; Jung, T. On the fractional $p$-Laplacian problems. J. Inequalities Appl. 2021. [CrossRef]

103. Fiscella, A. A fractional Kirchhoff problem involving a singular term and a critical nonlinearity. Adv. Nonlinear Anal. 2019, 8 , 645-660. [CrossRef]

104. Ji, C.; Fang, F.; Zhang, B. A multiplicity result for asymptotically linear Kirchhoff equations. Adv. Nonlinear Anal. 2019, 8, 267-277. [CrossRef]

105. Xiang, M.; Zhang, B.; Radulescu, V.D. Superlinear Schrodinger-Kirchhoff type problems involving the fractional $p-$ Laplacian and critical exponent. Adv. Nonlinear Anal. 2020, 9, 690-709. [CrossRef]

106. Zhikov, V.V. On some variational problems. Russ. J. Math. Phys. 1997, 5, 105-116.

107. Fan, X.; Zhao, D. On the spaces $L^{p(x)}(\Omega)$ and $W^{m, p(x)}(\Omega)$. J. Math. Anal. Appl. 2001, 263, 424-446. [CrossRef]

108. Kovácik, O.; Rákosnik, J. On spaces $L^{p(x)}$ and $W^{1, p(x)}$. Czech. Math. J. 1991, 41, 592-618.

109. Salort, A.M. A fractional Orlicz-Sobolev eigenvalue problem and related Hardy inequalities. arXiv 2018, arXiv:1807.03209.

110. Azroul, E.; Benkirane, A.; Srati, M. Existence of solutions for a nonlocal type problem in fractional Orlicz-Sobolev spaces. Adv. Oper. Theory 2020, 5, 1350-1375. [CrossRef]

111. Bott, R. Nondegenerate critical manifolds. Ann. Math. 1954, 60, 248-261. [CrossRef] 